\title{
The Borsuk-Ulam property for homotopy classes of maps between the torus and the Klein bottle
}

\author{
DACIBERG LIMA GONÇALVES * JOHN GUASCHI ${ }^{\dagger}$ \\ VINICIUS CASTELUBER LAASS $\ddagger$
}

\author{
17th November 2019
}

\begin{abstract}
Let $M$ be a topological space that admits a free involution $\tau$, and let $N$ be a topological space. A homotopy class $\beta \in[M, N]$ is said to have the Borsuk-Ulam property with respect to $\tau$ if for every representative map $f: M \rightarrow N$ of $\beta$, there exists a point $x \in M$ such that $f(\tau(x))=f(x)$. In this paper, we determine the homotopy classes of maps from the 2-torus $\mathbb{T}^{2}$ to the Klein bottle $\mathbb{K}^{2}$ that possess the Borsuk-Ulam property with respect to a free involution $\tau_{1}$ of $\mathbb{T}^{2}$ for which the orbit space is $\mathbb{T}^{2}$. Our results are given in terms of a certain family of homomorphisms involving the fundamental groups of $\mathbb{T}^{2}$ and $\mathbb{K}^{2}$.
\end{abstract}

\section{Introduction}

In the early twentieth century, St. Ulam conjectured that if $f: \mathbb{S}^{n} \rightarrow \mathbb{R}^{n}$ is a continuous map, there exists $x \in \mathbb{S}^{n}$ such that $f(A(x))=f(x)$, where $A: \mathbb{S}^{n} \rightarrow \mathbb{S}^{n}$ is the antipodal map. The confirmation of this result by K. Borsuk in 1933 [1], known as the Borsuk-Ulam theorem, was the beginning of what it now referred to as Borsuk-Ulam type theorems or the Borsuk-Ulam property. More information about the history and some applications of the Borsuk-Ulam theorem may be found in [9], for example.

One possible generalisation of the classical Borsuk-Ulam theorem is to substitute $\mathbb{S}^{n}$ and $\mathbb{R}^{n}$ by other spaces, and to replace the antipodal map by a free involution. A natural question is the following: does every continuous map collapse an orbit of the involution? More precisely, given topological spaces $M$ and $N$ such that $M$ admits a free involution $\tau$, we say that the triple $(M, \tau ; N)$ has the Borsuk-Ulam property if for every continuous map $f: M \rightarrow N$, there exists a point $x \in M$ such that $f(\tau(x))=f(x)$. For the cases where $M$ is a compact surface without boundary admitting a free involution $\tau$ and $N$ is either $\mathbb{R}^{2}$ or a compact surface without boundary, the triples $(M, \tau ; N)$ that have the Borsuk-Ulam property have been classified (see [3] and [4]). One generalisation of this property is to consider a local Borsuk-Ulam problem in the sense of the following definition: a homotopy class $\beta \in[M, N]$ has the Borsuk-Ulam property with respect to $\tau$ if for every representative $f: M \rightarrow N$ of $\beta$, there exists a point $x \in M$ such that $f(\tau(x))=f(x)$.

${ }^{*}$ Departamento de Matemática, IME, Universidade de São Paulo, Caixa Postal 66281, Ag. Cidade de São Paulo, CEP: 05314-970, São Paulo, SP, Brazil. e-mail: dlgoncal@ime.usp.br

${ }^{\dagger}$ Normandie Univ., UNICAEN, CNRS, Laboratoire de Mathématiques Nicolas Oresme UMR CNRS 6139, 14000 Caen, France. e-mail: john.guaschi@unicaen.fr

‡Departamento de Matemática, IME, Universidade Federal da Bahia, Av. Adhemar de Barros, S/N Ondina CEP: 40170-110, Salvador, BA, Brazil. e-mail: vinicius.laass@ufba.br 
In [5], the Borsuk-Ulam problem for homotopy classes of maps between compact surfaces without boundary was studied, and the sets $\left[\mathbb{T}^{2}, \mathbb{T}^{2}\right]$ and $\left[\mathbb{K}^{2}, \mathbb{K}^{2}\right]$ whose elements possess the Borsuk-Ulam property were characterised. By [4, Theorem 12], for any involution $\tau: \mathbb{T}^{2} \rightarrow \mathbb{T}^{2}$, the triple $\left(\mathbb{T}^{2}, \tau ; \mathbb{K}^{2}\right)$ does not have the Borsuk-Ulam property. Using this information, in this paper we classify the homotopy classes of maps from $\mathbb{T}^{2}$ to $\mathbb{K}^{2}$ that have the Borsuk-Ulam property for the orientation-preserving free involution $\tau_{1}$ of $\mathbb{T}^{2}$. Our approach, which we now describe, is similar to that used in [5]. First, as in [5, Theorems 12 and 19], we identify $\pi_{1}\left(\mathbb{T}^{2}, *\right)$ and $\pi_{1}\left(\mathbb{K}^{2}, *\right)$ with the free Abelian group $\mathbb{Z} \oplus \mathbb{Z}$ and the (non-trivial) semi-direct product $\mathbb{Z} \rtimes \mathbb{Z}$ respectively. These identifications will be helpful in formulating the results and in making explicit computations.

To prove our results, we will make use of the following algebraic description given in $[6$, Corollary 2.1] of the set $\left[\mathbb{T}^{2}, \mathbb{K}^{2}\right]$ in terms of pointed homotopy classes and the corresponding fundamental groups.

Proposition 1.1. The set $\left[\mathbb{T}^{2}, \mathbb{K}^{2}\right]$ is in bijection with the subset of $\operatorname{Hom}(\mathbb{Z} \oplus \mathbb{Z}, \mathbb{Z} \rtimes \mathbb{Z})$ whose elements are described as follows:

$$
\begin{array}{ll}
\text { Type 1: } \begin{cases}(1,0) \mapsto\left(i, 2 s_{1}+1\right) \\
(0,1) \mapsto\left(0,2 s_{2}\right)\end{cases} & \text { Type 3: }\left\{\begin{array}{l}
(1,0) \mapsto\left(0,2 s_{1}\right) \\
(0,1) \mapsto\left(i, 2 s_{2}+1\right)
\end{array}\right. \\
\text { Type 2: } \begin{cases}(1,0) \mapsto\left(i, 2 s_{1}+1\right) \\
(0,1) \mapsto\left(i, 2 s_{2}+1\right)\end{cases} & \text { Type 4: }\left\{\begin{array}{l}
(1,0) \mapsto\left(r_{1}, 2 s_{1}\right) \\
(0,1) \mapsto\left(r_{2}, 2 s_{2}\right),
\end{array}\right.
\end{array}
$$

where $i \in\{0,1\}$ and $s_{1}, s_{2} \in \mathbb{Z}$ for Types 1,2 and 3, and $r_{1}, r_{2}, s_{1}, s_{2} \in \mathbb{Z}$ and $r_{1} \geq 0$ for Type 4 .

Remark 1.2. The bijection of Proposition 1.1 may be obtained using standard arguments in homotopy theory that are described in detail in [11, Chapter V, Corollary 4.4], and more briefly in $[5$, Theorem 4]. In our specific case, the bijection is defined as follows: given a homotopy class $\beta \in\left[\mathbb{T}^{2}, \mathbb{K}^{2}\right]$, there exists a pointed map $f:\left(\mathbb{T}^{2}, *\right) \rightarrow\left(\mathbb{K}^{2}, *\right)$ that gives rise to a representative of $\beta$ if we omit the basepoints. The induced homomorphism $f_{\#}: \pi_{1}\left(\mathbb{T}^{2}, *\right) \rightarrow \pi_{1}\left(\mathbb{K}^{2}, *\right)$ is conjugate to exactly one of the elements of $\operatorname{Hom}(\mathbb{Z} \oplus \mathbb{Z}, \mathbb{Z} \rtimes \mathbb{Z})$ described in Proposition 1.1, which we denote by $\beta_{\#}$. Note that $\beta_{\#}$ does not depend on the choice of $f$.

In order to solve the Borsuk-Ulam problem for homotopy classes, we now describe the relevant involution of $\mathbb{T}^{2}$. Consider the following short exact sequence:

$$
1 \rightarrow \pi_{1}\left(\mathbb{T}^{2}\right)=\mathbb{Z} \oplus \mathbb{Z} \stackrel{i_{1}}{\longrightarrow} \pi_{1}\left(\mathbb{T}^{2}\right)=\mathbb{Z} \oplus \mathbb{Z} \stackrel{\theta_{1}}{\longrightarrow} \mathbb{Z}_{2} \rightarrow 1
$$

where:

$$
i_{1}:\left\{\begin{array}{l}
(1,0) \longmapsto(2,0) \\
(0,1) \longmapsto(0,1)
\end{array} \quad \theta_{1}:\left\{\begin{array}{l}
(1,0) \longmapsto \overline{1} \\
(0,1) \longmapsto \overline{0}
\end{array}\right.\right.
$$

By standard results in covering space theory, there exists a double covering $c_{1}: \mathbb{T}^{2} \rightarrow \mathbb{T}^{2}$ whose induced homomorphism on the level of fundamental groups is $i_{1}$. If $\tau_{1}: \mathbb{T}^{2} \rightarrow \mathbb{T}^{2}$ is the nontrivial deck transformation associated with $c_{1}$, then $\tau_{1}$ is a free involution. Further, $\tau_{1}$ lifts to a homeomorphism $\widehat{\tau}_{1}: \mathbb{R}^{2} \rightarrow \mathbb{R}^{2}$, where $\widehat{\tau}_{1}(x, y)=\left(x+\frac{1}{2}, y\right)$ for all $(x, y) \in \mathbb{R}^{2}$. In this paper, we classify the elements of the set $\left[\mathbb{T}^{2}, \mathbb{K}^{2}\right]$ that possess the Borsuk-Ulam property with respect to $\tau_{1}$. This is achieved in the following theorem, which is the main result of this paper.

Theorem 1.3. Given a non-zero integer $t$, let $e(t)$ be its 2-adic evaluation. With the notation of Proposition 1.1, let $\beta \in\left[\mathbb{T}^{2}, \mathbb{K}^{2}\right]$ and $\beta_{\#} \in \operatorname{Hom}(\mathbb{Z} \oplus \mathbb{Z}, \mathbb{Z} \rtimes \mathbb{Z})$. Then $\beta$ has the Borsuk-Ulam property with respect to $\tau_{1}$ if and only if one of the following conditions is satisfied: 
(a) $\beta_{\#}$ is a homomorphism of Type 3 .

(b) $\beta_{\#}$ is a homomorphism of Type 4, where $s_{1}$ is odd and $r_{2} \neq 0$, and additionally $e\left(r_{1}\right)>e\left(r_{2}\right)$ if $r_{1} \neq 0$.

Besides the introduction and an Appendix, this paper consists of three sections. In Section 2, we show how to reduce the number of homotopy classes to be studied with respect to the BorsukUlam property. In Section 3, we study the normal closure of $\sigma^{2}$ in $P_{2}\left(\mathbb{K}^{2}\right)$, which is a free group of infinite rank. A convenient basis for this subgroup is obtained in the Appendix. In Section 4, we prove Theorem 1.3.

The study of the free involution $\tau_{2}$ of $\mathbb{T}^{2}$ for which the associated orbit space is the Klein bottle is the subject of work in progress.

\section{Some preliminary results}

The following results will enable us to reduce the number of cases to be analysed in the proof of Theorems 1.3.

Lemma 2.1. Let $M$ and $N$ be topological spaces, let $\tau: M \rightarrow M$ be a free involution, and let $H: N \rightarrow N$ be a homeomorphism. Then the map $\mathcal{H}:[M, N] \rightarrow[M, N]$ defined by $\mathcal{H}([f])=[H \circ f]$ for all maps $f: M \rightarrow N$ is a bijection. Further, if $\beta \in[M, N]$ is a homotopy class, then $\beta$ has the Borsuk-Ulam property with respect to $\tau$ if and only if $\mathcal{H}(\beta)$ has the Borsuk-Ulam property with respect to $\tau$.

Proof. Clearly the map $\mathcal{H}$ is a bijection whose inverse is given by $\mathcal{H}^{-1}([g])=\left[H^{-1} \circ g\right]$. To prove the second part of the statement, let $\beta \in[M, N]$ be a homotopy class that has the Borsuk-Ulam property with respect to $\tau$, and let $g \in \mathcal{H}(\beta)$. Thus $H^{-1} \circ g \in \beta$, and hence there exists $x \in M$ such that $H^{-1} \circ g(x)=H^{-1} \circ g(\tau(x))$. Therefore $g(x)=g(\tau(x))$, and we conclude that $\mathcal{H}(\beta)$ has the Borsuk-Ulam property. The converse follows in a similar manner using $H^{-1}$.

Proposition 2.2. Let $\tau: \mathbb{T}^{2} \rightarrow \mathbb{T}^{2}$ be a free involution, and let $\beta, \beta^{\prime} \in\left[\mathbb{T}^{2} ; \mathbb{K}^{2}\right]$ such that $\beta_{\#}, \beta_{\#}^{\prime}$ are both of Type 1, 2 or 3. Suppose that the second coordinates of $\beta_{\#}(\omega)$ and $\beta_{\#}^{\prime}(\omega)$ are equal for all $\omega \in \pi_{1}\left(\mathbb{T}^{2}, *\right)$ and the integer $i$ that defines the homomorphism $\beta_{\#}$ (resp. $\beta_{\#}^{\prime \prime}$ ) is equal to 0 (resp. 1). Then $\beta$ has the Borsuk-Ulam property with respect to $\tau$ if and only if $\beta^{\prime}$ does.

Proof. Let $h: \mathbb{Z} \rtimes \mathbb{Z} \rightarrow \mathbb{Z} \rtimes \mathbb{Z}$ be the homomorphism defined on the generators of $\mathbb{Z} \rtimes \mathbb{Z}$ by $h(1,0)=(1,0)$ and $h(0,1)=(1,1)$. Then $h$ is well defined, and it is an isomorphism whose inverse $h^{-1}: \mathbb{Z} \rtimes \mathbb{Z} \rightarrow \mathbb{Z} \rtimes \mathbb{Z}$ is given by $h^{-1}(1,0)=(1,0)$ and $h^{-1}(0,1)=(-1,1)$. By $[12$, Theorem 5.6.2], there exists a homeomorphism $H:\left(\mathbb{K}^{2}, *\right) \rightarrow\left(\mathbb{K}^{2}, *\right)$ such that $H_{\#}=h$ and $H_{\#}^{-1}=h^{-1}$. Suppose that $\beta_{\#}$ and $\beta_{\#}^{\prime}$ are both of Type 1 , and that they satisfy the hypothesis of the statement, and let $f:\left(\mathbb{T}^{2}, *\right) \rightarrow\left(\mathbb{K}^{2}, *\right)$ be a representative map of $\beta$. Without loss of generality, we may suppose that $f_{\#}=\beta_{\#}$. Assume that $\beta$ has the Borsuk-Ulam property with respect to $\tau$. Then:

$$
\begin{aligned}
(H \circ f)_{\#}(1,0) & =h\left(\beta_{\#}(1,0)\right)=h\left(0,2 s_{1}+1\right)=h(0,1)^{2 s_{1}+1} \\
& =((1,1)(1,1))^{s_{1}}(1,1)=(0,2)^{s_{1}}(1,1)=\left(1,2 s_{1}+1\right), \text { and } \\
(H \circ f)_{\#}(0,1) & =h\left(\beta_{\#}(0,1)\right)=h\left(0,2 s_{2}\right)=h(0,1)^{2 s_{2}}=((1,1)(1,1))^{s_{2}}=(0,2)^{s_{2}}=\left(0,2 s_{2}\right) .
\end{aligned}
$$

Then $H \circ f$ is a representative of $\beta^{\prime}$, and the conclusion follows from Lemma 2.1. The converse follows in a similar way using $H^{-1}$ instead $H$. The arguments for the cases of homomorphisms of Types 2 and 3 are analogous, and the details are left to the reader.

Remark 2.3. Using Lemma 2.1, one may show that Proposition 2.2 holds in more generality. However the above statement will be sufficient for our purposes. 


\section{The normal closure of $\sigma^{2}$ in $P_{2}\left(\mathbb{K}^{2}\right)$}

Let $S$ be a compact, connected surface without boundary. The ordered 2-point configuration space of $S$ is defined by $F_{2}(S)=\{(x, y) \in S \times S \mid x \neq y\}, D_{2}(S)$ is the orbit space of $F_{2}(S)$ by the free involution $\tau_{S}: F_{2}(S) \rightarrow F_{2}(S)$ defined by $\tau_{S}(x, y)=(y, x)$, and $P_{2}(S)=\pi_{1}\left(F_{2}(S)\right)$ and $B_{2}(S)=\pi_{1}\left(D_{2}(S)\right)$ are the pure and full 2-string braid groups of $S$ respectively [2]. We have a short exact sequence:

$$
1 \rightarrow P_{2}(S) \rightarrow B_{2}(S) \stackrel{\pi}{\rightarrow} \mathbb{Z}_{2} \rightarrow 1
$$

where $\pi: B_{2}(S) \rightarrow \mathbb{Z}_{2}$ is the homomorphism that to an element of $B_{2}(S)$ associates its permutation. If $p_{1}: F_{2}(S) \rightarrow S$ is the projection onto the first coordinate, the map $\left(p_{1}\right)_{\#}: P_{2}(S) \rightarrow \pi_{1}(S)$ may be interpreted geometrically as the surjective homomorphism that forgets the second string. Let $\sigma \in B_{2}(S) \backslash P_{2}(S)$ be the Artin generator of $B_{2}(S)$ that geometrically swaps the two basepoints. Then $\sigma^{2} \in P_{2}(S)$, and the normal closure of $\sigma^{2}$ in $P_{2}(S)$, which we denote by $\overline{\left\langle\sigma^{2}\right\rangle}$, is also the normal closure of $\sigma^{2}$ in $B_{2}(S)$ by (2). In this section, we shall take $S$ to be the Klein bottle, and we will show that $\overline{\left\langle\sigma^{2}\right\rangle}$ is a free group of countably-infinite rank for which we shall exhibit a basis. We will also express certain elements of $\overline{\left\langle\sigma^{2}\right\rangle}$ in this basis.

The following proposition summarises some results of [5, Section 4] regarding the structure of $P_{2}\left(\mathbb{K}^{2}\right)$ and the action by conjugation by $\sigma$ on this group.

Proposition 3.1. [5, Theorems 19 and 20] The group $P_{2}\left(\mathbb{K}^{2}\right)$ is isomorphic to the semi-direct product $F(u, v) \rtimes_{\theta}(\mathbb{Z} \rtimes \mathbb{Z})$, where $F(u, v)$ is the free group of rank 2 on the set $\{u, v\}$ and the action $\theta: \mathbb{Z} \rtimes \mathbb{Z} \rightarrow \operatorname{Aut}(F(u, v))$ is defined as follows:

$$
\theta(m, n):\left\{\begin{array}{l}
u \mapsto B^{m-\delta_{n}} u^{\varepsilon_{n}} B^{-m+\delta_{n}} \\
v \mapsto B^{m} v u^{-2 m} B^{-m+\delta_{n}} \\
B \mapsto B^{\varepsilon_{n}}
\end{array}\right.
$$

where $\delta_{n}=\left\{\begin{array}{l}0 \quad \text { if } n \text { is even } \\ 1 \quad \text { if } n \text { is odd, }\end{array} \varepsilon_{n}=(-1)^{n}\right.$ and $B=u v u v^{-1}$. With respect to this description, the following properties hold:

- the element $\sigma \in B_{2}\left(\mathbb{K}^{2}\right)$ satisfies $\sigma^{2}=(B ; 0,0)$.

- if $l_{\sigma}: P_{2}\left(\mathbb{K}^{2}\right) \rightarrow P_{2}\left(\mathbb{K}^{2}\right)$ is the homomorphism defined by $l_{\sigma}(b)=\sigma b \sigma^{-1}$ for all $b \in P_{2}\left(\mathbb{K}^{2}\right)$, then:

$$
\begin{array}{rlrl}
l_{\sigma}\left(u^{r} ; 0,0\right) & =\left(\left(B u^{-1}\right)^{r} B^{-r} ; r, 0\right) & l_{\sigma}(\mathbf{1} ; m, 0) & =(\mathbf{1} ; m, 0) \\
l_{\sigma}\left(v^{s} ; 0,0\right) & =\left((u v)^{-s}(u B)^{\delta_{s}} ; 0, s\right) & l_{\sigma}(\mathbf{1} ; 0, n)=\left(B^{\delta_{n}} ; 0, n\right) \\
l_{\sigma}(B ; 0,0) & =(B ; 0,0) &
\end{array}
$$

for all $m, n, r, s \in \mathbb{Z}$, where the symbol 1 denotes the trivial element of $F(u, v)$.

- the homomorphism $\left(p_{1}\right)_{\#}: P_{2}\left(\mathbb{K}^{2}\right) \rightarrow \pi_{1}\left(\mathbb{K}^{2}\right)=\mathbb{Z} \rtimes \mathbb{Z}$ satisfies $\left(p_{1}\right)_{\#}(w ; r, s)=(r, s)$.

From now on, we identify $P_{2}\left(\mathbb{K}^{2}\right)$ with $F(u, v) \rtimes_{\theta}(\mathbb{Z} \rtimes \mathbb{Z})$ without further comment.

Remark 3.2. It follows from Proposition 3.1 that for all $m, n \in \mathbb{Z}$, the automorphism $\theta(m, n)$ : $F(u, v) \rightarrow F(u, v)$ depends only on $m$ and the parity of $n$, in particular $\theta(m, n)=\theta\left(m, \delta_{n}\right)$. 
Consider the following maps:

$$
\iota:\left\{\begin{aligned}
F(u, v) & \longrightarrow P_{2}\left(\mathbb{K}^{2}\right) \\
w & \longmapsto(w ; 0,0)
\end{aligned} \text { and } p_{F}:\left\{\begin{aligned}
P_{2}\left(\mathbb{K}^{2}\right) & \longrightarrow F(u, v) \\
(w ; m, n) & \longmapsto w .
\end{aligned}\right.\right.
$$

Note that $\iota$ is a homomorphism, but due to the action $\theta, p_{F}$ is not. Consider the map $\rho: F(u, v) \rightarrow$ $F(u, v)$ defined by:

$$
\rho=p_{F} \circ l_{\sigma} \circ \iota
$$

and the homomorphism $g: F(u, v) \rightarrow \mathbb{Z} \rtimes \mathbb{Z}$ defined on the basis $\{u, v\}$ by:

$$
\left\{\begin{array}{l}
g(u)=(1,0) \\
g(v)=(0,1)
\end{array}\right.
$$

Using Proposition 3.1 and (3), we obtain the following commutative diagram:

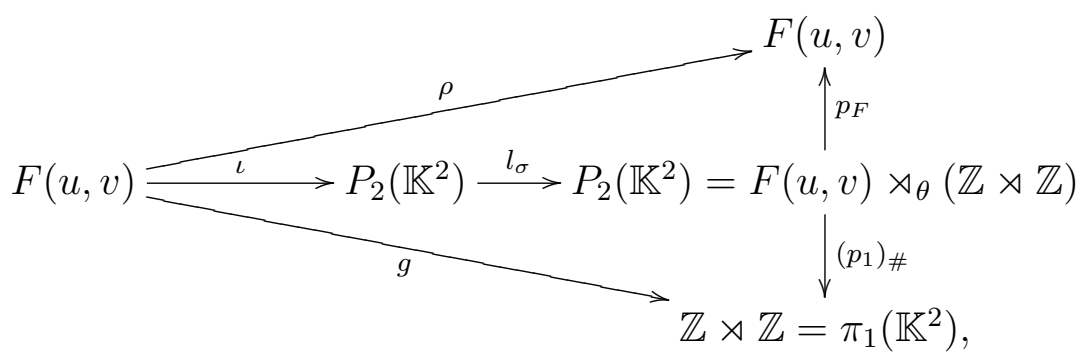

from which it follows that:

$$
l_{\sigma}(w ; 0,0)=(\rho(w) ; g(w))
$$

for all $w \in F(u, v)$. Further if $w, z \in F(u, v)$ then:

$$
\begin{aligned}
\rho(w z) & =\left(p_{F} \circ l_{\sigma}\right)(w z ; 0,0)=p_{F}\left(l_{\sigma}(w ; 0,0) \cdot l_{\sigma}(z ; 0,0)\right)=p_{F}((\rho(w) ; g(w)) \cdot(\rho(z) ; g(z))) \\
& =p_{F}(\rho(w) \theta(g(w))(\rho(z)) ; g(w) g(z))=\rho(w) \theta(g(w))(\rho(z)) .
\end{aligned}
$$

Thus the map $\rho: F(u, v) \rightarrow F(u, v)$ is not a homomorphism, but if $w \in \operatorname{Ker}(g)$ then:

$$
\rho(w z)=\rho(w) \rho(z) .
$$

In Theorem A.3 of the Appendix, we prove that:

$$
\operatorname{Ker}(g)=\left\langle B_{k, l}:=v^{k} u^{l} B u^{-l} v^{-k}, k, l \in \mathbb{Z} \mid-\right\rangle .
$$

Let $w \in F(u, v)$ and $(m, n) \in \mathbb{Z} \rtimes \mathbb{Z}$. Using Proposition 3.1, we see that $B \in \operatorname{Ker}(g)$, and then that:

$$
\theta(m, n)\left(w B w^{-1}\right)=\theta(m, n)(w) B^{\varepsilon_{n}} \theta(m, n)(w)^{-1} \in \operatorname{Ker}(g)
$$

Further,

$$
\begin{aligned}
l_{\sigma}\left(w B w^{-1} ; 0,0\right) & =l_{\sigma}(w ; 0,0) l_{\sigma}(B ; 0,0) l_{\sigma}(w ; 0,0)^{-1} \stackrel{(5)}{=}(\rho(w) ; g(w))(B ; 0,0)(\rho(w) ; g(w))^{-1} \\
& =(\rho(w) \theta(g(w))(B) ; g(w))\left(\theta\left(g(w)^{-1}\right)\left(\rho(w)^{-1}\right) ; g(w)^{-1}\right) \\
& =\left(\rho(w) \theta(g(w))(B) \rho(w)^{-1} ; 0,0\right) .
\end{aligned}
$$


Composing (10) by $p_{F}$, it follows from (5) and (9) that:

$$
\rho\left(w B w^{-1}\right)=\rho(w) \theta(g(w))(B) \rho(w)^{-1} \in \operatorname{Ker}(g) .
$$

In particular, $\rho\left(B_{k, l}\right) \in \operatorname{Ker}(g)$ for all $k, l \in \mathbb{Z}$, and using (7) and (8), the restriction of $\rho$ to $\operatorname{Ker}(g)$ yields an endomorphism of $\operatorname{Ker}(g)$, which we also denote by $\rho$. Further, $\theta(m, n)\left(B_{k, l}\right) \in$ $\operatorname{Ker}(g)$ for all $k, l, m, n \in \mathbb{Z}$ using (9), and thus $\theta$ determines a homomorphism from $\mathbb{Z} \rtimes \mathbb{Z}$ to $\operatorname{Aut}(\operatorname{Ker}(g))$, which we also denote by $\theta$. Note that for all $m, m \in \mathbb{Z}$, the endomorphism $\theta(m, n): \operatorname{Ker}(g) \rightarrow \operatorname{Ker}(g)$ is indeed surjective. To see this, let $k, l \in \mathbb{Z}$, and let $\xi \in F(u, v)$ be such that $\theta(m, n)(\xi)=v^{k} u^{l}$. Then $\theta(m, n)\left(\xi B^{\varepsilon_{n}} \xi^{-1}\right)=B_{k, l}$, and $\xi B^{\varepsilon_{n}} \xi^{-1} \in \operatorname{Ker}(g)$ because $B \in \operatorname{Ker}(g)$. Hence the image of $\theta(m, n): \operatorname{Ker}(g) \rightarrow \operatorname{Ker}(g)$ contains the basis $\left\{B_{k, l}\right\}_{k, l \in \mathbb{Z}}$ of $\operatorname{Ker}(g)$, and so this homomorphism is surjective.

The following result describes the subgroup $\overline{\left\langle\sigma^{2}\right\rangle}$.

Proposition 3.3. The injective homomorphism $\iota: F(u, v) \rightarrow P_{2}\left(\mathbb{K}^{2}\right)$ defined by $\iota(w)=(w ; 0,0)$ for all $w \in F(u, v)$, restricts to an isomorphism between $\operatorname{Ker}(g)$ and $\overline{\left\langle\sigma^{2}\right\rangle}$, which we also denote by ८. In particular, $\overline{\left\langle\sigma^{2}\right\rangle}$ is a free group of infinite rank for which a basis is given by $\left\{\left(B_{k, l} ; 0,0\right)\right\}_{k, l \in \mathbb{Z}}$, and $l_{\sigma}\left(\overline{\left\langle\sigma^{2}\right\rangle}\right) \subset \operatorname{Ker}\left(\left(p_{1}\right)_{\#}\right)$. Up to this isomorphism, the homomorphisms $\theta: \mathbb{Z} \rtimes \mathbb{Z} \rightarrow \operatorname{Aut}(\operatorname{Ker}(g))$ and $\rho: \operatorname{Ker}(g) \rightarrow \operatorname{Ker}(g)$ induce homomorphisms $\mathbb{Z} \rtimes \mathbb{Z} \rightarrow \operatorname{Aut}\left(\overline{\left\langle\sigma^{2}\right\rangle}\right)$ and $\overline{\left\langle\sigma^{2}\right\rangle} \rightarrow \overline{\left\langle\sigma^{2}\right\rangle}$, which we also denote by $\theta$ and $\rho$ respectively. Further, the following diagram is commutative:

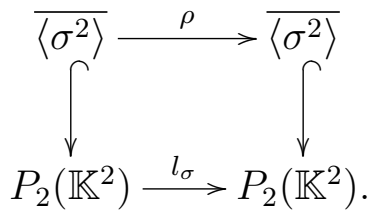

Proof. Let $H=\iota(\operatorname{Ker}(g)) \subset P_{2}\left(\mathbb{K}^{2}\right)$. We start by showing that $\overline{\left\langle\sigma^{2}\right\rangle}=H$. To see this, first note that $\sigma^{2}=(B ; 0,0)$ and $\iota\left(B_{k, l}\right) \in \overline{\left\langle\sigma^{2}\right\rangle}$ for all $k, l \in \mathbb{Z}$, hence $H \subset \overline{\left\langle\sigma^{2}\right\rangle}$ by (8). Conversely, for all $w \in F(u, v), q \in \mathbb{Z} \rtimes \mathbb{Z}$ and $k, l \in \mathbb{Z}$, we have:

$$
(w ; q)\left(B_{k, l} ; 0,0\right)(w ; q)^{-1}=\left(w \theta(q)\left(B_{k, l}\right) ; q\right)\left(\theta\left(q^{-1}\right)\left(w^{-1}\right) ; q^{-1}\right)=\left(w \theta(q)\left(B_{k, l}\right) w^{-1} ; 0,0\right) .
$$

Since $\theta(q)\left(B_{k, l}\right) \in \operatorname{Ker}(g)$ and $\operatorname{Ker}(g)$ is normal in $F(u, v)$, it follows that $w \theta(q)\left(B_{k, l}\right) w^{-1} \in$ $\operatorname{Ker}(g)$, and so $(w ; q)\left(B_{k, l} ; 0,0\right)(w ; q)^{-1} \in \iota(\operatorname{Ker}(g))=H$ by (12). Hence $H$ is a normal subgroup of $P_{2}\left(\mathbb{K}^{2}\right)$ by (8), and since $\sigma^{2} \in H$, we conclude that $\overline{\left\langle\sigma^{2}\right\rangle} \subset H$. Thus $\overline{\left\langle\sigma^{2}\right\rangle}=H$ as required. Since $\iota$ is injective, the induced homomorphism $\iota: \operatorname{Ker}(g) \rightarrow \overline{\left\langle\sigma^{2}\right\rangle}$ is an isomorphism, and the image of the basis $\left\{B_{k, l}\right\}_{k, l \in \mathbb{Z}}$ by $\iota$ yields a basis of the free group $\overline{\left\langle\sigma^{2}\right\rangle}$. The commutativity of the given diagram follows by considering the images of the elements of this basis $\left\{\left(B_{k, l} ; 0,0\right)\right\}_{k, l \in \mathbb{Z}}$ and using (10).

Remark 3.4. Although $\operatorname{Ker}(g)$ and $\overline{\left\langle\sigma^{2}\right\rangle}$ are isomorphic by Proposition 3.3, our results will be stated in terms of $\overline{\left\langle\sigma^{2}\right\rangle}$, since we will formulate most of our equations in this group.

The following result provides a normal form for elements of $F(u, v)$ in terms of $g$ and $\overline{\left\langle\sigma^{2}\right\rangle}$.

Proposition 3.5. Let $w \in F(u, v)$, and let $g(w)=(r, s)$. Then there exists a unique element $x \in \overline{\left\langle\sigma^{2}\right\rangle}$ such that $w=u^{r} v^{s} x$.

Proof. Let $w$ be as in the statement, and let $x=v^{-s} u^{-r} w$. Then $w=u^{r} v^{s} x$, and:

$$
g(x)=g\left(v^{-s} u^{-r} w\right)=g(v)^{-s} g(u)^{-r} g(w)=(0,-s)(-r, 0)(r, s)=(0,-s)(0, s)=(0,0) .
$$

So $x \in \overline{\left\langle\sigma^{2}\right\rangle}$. Clearly $x$ is unique. 
Let $p, q \in \mathbb{Z}$. Since $\overline{\left\langle\sigma^{2}\right\rangle}$ is a normal subgroup of $F(u, v)$, the following homomorphism is well defined:

$$
\begin{aligned}
c_{p, q}: \overline{\left\langle\sigma^{2}\right\rangle} & \longrightarrow \overline{\left\langle\sigma^{2}\right\rangle} \\
x & \longmapsto v^{p} u^{q} x u^{-q} v^{-p} .
\end{aligned}
$$

Lemma 3.6. Let $k, l, p, q \in \mathbb{Z}$, and let $(m, n) \in \mathbb{Z} \rtimes \mathbb{Z}$. With the notation of Proposition 3.1, there exist $\gamma, \lambda, \eta \in \overline{\left\langle\sigma^{2}\right\rangle}$ such that:

(a) $\theta(m, n)\left(B_{k, l}\right)=\gamma B_{k, \varepsilon_{n} l-2 \delta_{k} m}^{\varepsilon_{n}} \gamma^{-1}$.

(b) $\rho\left(B_{k, l}\right)=\lambda B_{-k, \varepsilon_{(k+1)} \varepsilon^{\varepsilon_{k}}} \lambda^{-1}$.

(c) $c_{p, q}\left(B_{k, l}\right)=\eta B_{k+p, l+\varepsilon_{k} q} \eta^{-1}$.

Proof. During the proof, we will make use freely of Proposition 3.1. First, we have:

$$
\theta(m, n)\left(B_{k, l}\right)=\theta(m, n)\left(v^{k} u^{l} B u^{-l} v^{-k}\right)=\theta(m, n)\left(v^{k} u^{l}\right) B^{\varepsilon_{n}} \theta(m, n)\left(v^{k} u^{l}\right)^{-1}=\gamma B_{k, \varepsilon_{n} l-2 \delta_{k} m}^{\varepsilon_{n}} \gamma^{-1},
$$

where $\gamma=\theta(m, n)\left(v^{k} u^{l}\right) u^{\varepsilon_{n+1} l+2 \delta_{k} m} v^{-k} \in F(u, v)$. To complete the proof of item (a), it remains to show that $\gamma \in \operatorname{Ker}(g)$. Since:

$$
\gamma=\left(B^{m} v u^{-2 m} B^{-m+\delta_{n}}\right)^{k}\left(B^{m-\delta_{n}} u^{\varepsilon_{n}} B^{-m+\delta_{n}}\right)^{l} u^{\varepsilon_{n+1} l+2 \delta_{k} m} v^{-k},
$$

and $B \in \operatorname{Ker}(g)$, it follows that:

$$
\begin{aligned}
g(\gamma) & =((0,1)(-2 m, 0))^{k}\left(\varepsilon_{n} l, 0\right)\left(\varepsilon_{n+1} l+2 \delta_{k} m, 0\right)(0,-k)=(2 m, 1)^{k}\left(\varepsilon_{n} l+\varepsilon_{n+1} l+2 \delta_{k} m,-k\right) \\
& =\left(2 \delta_{k} m, k\right)\left(2 \delta_{k} m,-k\right)=\left(2 \delta_{k} m+2 \delta_{k} \varepsilon_{k} m, 0\right)=(0,0),
\end{aligned}
$$

using the fact that $\varepsilon_{k}=-1$ if $k$ is odd, and $\delta_{k}=0$ if $k$ is even. Hence $\gamma \in \operatorname{Ker}(g)$. To prove item (b), first note that:

$$
\theta\left(g\left(v^{k} u^{l}\right)\right)(B)=\theta((0, k)(l, 0))(B)=\theta\left(\varepsilon_{k} l, k\right)(B)=B^{\varepsilon_{k}} .
$$

By (11) and (14), we have:

$$
\rho\left(B_{k, l}\right)=\rho\left(v^{k} u^{l} B u^{-l} v^{-k}\right)=\rho\left(v^{k} u^{l}\right) \theta\left(g\left(v^{k} u^{l}\right)\right)(B) \rho\left(v^{k} u^{l}\right)^{-1}=\lambda B_{-k, \varepsilon_{(k+1)}{ }^{\varepsilon_{k}} \lambda^{-1}},
$$

where $\lambda=\rho\left(v^{k} u^{l}\right) u^{\varepsilon_{k} l} v^{k} \in F(u, v)$. It remains to show that $\lambda \in \operatorname{Ker}(g)$. We have:

$$
\begin{aligned}
g(\lambda) & =g\left(\rho\left(v^{k} u^{l}\right) u^{\varepsilon_{k} l} v^{k}\right)=g\left(\left(p_{F} \circ l_{\sigma} \circ i\right)\left(v^{k} u^{l}\right)\right) \cdot\left(\varepsilon_{k} l, k\right) \\
& =g\left(p_{F}\left(\left((u v)^{-k}(u B)^{\delta_{k}} ; 0, k\right)\left(\left(B u^{-1}\right)^{l} B^{-l} ; l, 0\right)\right)\right) \cdot\left(\varepsilon_{k} l, k\right) \\
& =g\left((u v)^{-k}(u B)^{\delta_{k}} \theta(0, k)\left(\left(B u^{-1}\right)^{l} B^{-l}\right)\right) \cdot\left(\varepsilon_{k} l, k\right) \\
& =(1,1)^{-k}\left(\delta_{k}, 0\right) \cdot g\left(\left(B^{\varepsilon_{k}}\left(B^{-\delta_{k}} u^{\varepsilon_{k}} B^{\delta_{k}}\right)^{-1}\right)^{l} B^{-\varepsilon_{k} l}\right) \cdot\left(\varepsilon_{k} l, k\right) \\
& =\left(\delta_{-k},-k\right)\left(\delta_{k}, 0\right)\left(-\varepsilon_{k} l, 0\right)\left(\varepsilon_{k} l, k\right)=\left(\delta_{-k}+\varepsilon_{-k} \delta_{k}, 0\right)=(0,0),
\end{aligned}
$$

since $\delta_{k}=0$ if $k$ is even, and $\varepsilon_{k}=-1$ if $k$ is odd. Hence $\lambda \in \operatorname{Ker}(g)$. Finally we prove item (c). We have:

$$
c_{p, q}\left(B_{k, l}\right)=v^{p} u^{q} B_{k, l} u^{-q} v^{-p}=v^{p} u^{q} v^{k} u^{l} B u^{-l} v^{-k} u^{-q} v^{-p}=\eta B_{k+p, l+\varepsilon_{k} q} \eta^{-1},
$$

where $\eta=v^{p} u^{q} v^{k} u^{\varepsilon_{k+1} q} v^{-k-p} \in F(u, v)$. It remains to show that $\eta \in \operatorname{Ker}(g)$. This is indeed the case because:

$$
g(\eta)=(0, p)(q, k)\left(\varepsilon_{k+1} q,-k-p\right)=(0, p)\left(q+\varepsilon_{k} \varepsilon_{k+1} q,-p\right)=(0, p)(0,-p)=(0,0),
$$

which completes the proof. 
Let ${\overline{\left\langle\sigma^{2}\right\rangle}}_{\mathrm{Ab}}$ denote the Abelianisation of the group $\overline{\left\langle\sigma^{2}\right\rangle}$. By abuse of notation, for all $k, l \in \mathbb{Z}$, we denote the image of a generator $B_{k, l}$ in ${\overline{\left\langle\sigma^{2}\right\rangle}}_{\mathrm{Ab}}$ by $B_{k, l}$. By Proposition 3.3, ${\overline{\left\langle\sigma^{2}\right\rangle}}_{\mathrm{Ab}}$ is the free Abelian group for which $\left\{B_{k, l}:=v^{k} u^{l} B u^{-l} v^{-k} \mid k, l \in \mathbb{Z}\right\}$ is a basis, namely:

$$
{\overline{\left\langle\sigma^{2}\right\rangle}}_{\mathrm{Ab}}=\bigoplus_{k, l \in \mathbb{Z}} \mathbb{Z}\left[B_{k, l}\right]
$$

For all $(m, n) \in \mathbb{Z} \rtimes \mathbb{Z}$ and $p, q \in \mathbb{Z}$, the endomorphisms $\theta(m, n), \rho$ and $\left(c_{p, q}\right)$ of $\overline{\left\langle\sigma^{2}\right\rangle}$ induce endomorphisms $\theta(m, n)_{\mathrm{Ab}}, \rho_{\mathrm{Ab}}$ and $\left(c_{p, q}\right)_{\mathrm{Ab}}$ of ${\overline{\left\langle\sigma^{2}\right\rangle}}_{\mathrm{Ab}}$ respectively, and by Lemma 3.6, for all $k, l \in \mathbb{Z}$, they satisfy:

$$
\begin{aligned}
\theta(m, n)_{\mathrm{Ab}}\left(B_{k, l}\right) & =\varepsilon_{n} B_{k, \varepsilon_{n} l-2 \delta_{k} m} \\
\rho_{\mathrm{Ab}}\left(B_{k . l}\right) & =\varepsilon_{k} B_{-k, \varepsilon_{(k+1)} l}, \text { and } \\
\left(c_{p, q}\right)_{\mathrm{Ab}}\left(B_{k, l}\right) & =B_{k+p, l+\varepsilon_{k} q} .
\end{aligned}
$$

Let $k, l \in \mathbb{Z}$ and $r \in\{0,1\}$. If $x$ and $y$ are elements of a group, let $[x, y]=x y x^{-1} y^{-1}$ denote their commutator. In the rest of the paper, we will be particularly interested in the following elements of $F(u, v)$ :

(I) $T_{k, r}=u^{k}\left(B^{\varepsilon_{r}} u^{-\varepsilon_{r}}\right)^{k \varepsilon_{r}}$.

(II) $I_{k}=v^{k}(v B)^{-k}$.

(III) $O_{k, l}=\left[v^{2 k}, u^{l}\right]$.

(IV) $J_{k, l}=v^{2 k}\left(v u^{l}\right)^{-2 k}$.

As we shall now see, $T_{k, r}, I_{k}, O_{k, l}$ and $J_{k, l}$ are elements of $\overline{\left\langle\sigma^{2}\right\rangle}$, and their projections into $\overline{\left\langle\sigma^{2}\right\rangle}$ Ab will be denoted by $\widetilde{T}_{k, r}, \widetilde{I}_{k}, \widetilde{O}_{k, l}$ and $\widetilde{J}_{k, l}$ respectively. The following result describes the decomposition of these Abelianisations in terms of the basis $\left\{B_{k, l}\right\}_{k, l}$. If $l \in \mathbb{Z}$, let $\sigma_{l}$ denote its sign, i.e $\sigma_{l}=1$ if $l>0, \sigma_{l}=-1$ if $l<0$, and $\sigma_{l}=0$ if $l=0$.

Proposition 3.7. Let $k, l \in \mathbb{Z}$ and $r \in\{0,1\}$.

(a) The elements $T_{k, r}, I_{k}, O_{k, l}$ and $J_{k, l}$ belong to $\overline{\left\langle\sigma^{2}\right\rangle}$.

(b) If $k=0$ then $\widetilde{T}_{0, r}=\widetilde{I}_{0}=0$, and if $k=0$ or $l=0$ then $\widetilde{O}_{k, l}=\widetilde{J}_{k, l}=0$.

(c) For all $k, l \neq 0$ :

$$
\begin{aligned}
\widetilde{T}_{k, r} & =\sigma_{k} \sum_{i=1}^{\sigma_{k} k} B_{0, \sigma_{k}\left(i+\left(\sigma_{k}(1-2 r)-1\right) / 2\right)} \\
\widetilde{I}_{k} & =-\sigma_{k} \sum_{i=1}^{\sigma_{k} k} B_{\sigma_{k} i+\left(1-\sigma_{k}\right) / 2,0} \\
\widetilde{J}_{k, l} & =-\sigma_{k} \sigma_{l} \sum_{i=1}^{\sigma_{k} k} \sum_{j=1}^{\sigma_{l} l} B_{\sigma_{k}(2 i-1), \sigma_{l}\left(j-\left(1+\sigma_{l}\right) / 2\right)} \\
\widetilde{O}_{k, l} & =\sigma_{k} \sigma_{l} \sum_{i=1}^{\sigma_{k} k} \sum_{j=1}^{\sigma_{l} l}\left(B_{\sigma_{k}(2 i-1),-\sigma_{l} j+\left(\sigma_{l}-1\right) / 2}-B_{\sigma_{k}(2 i-1)-1, \sigma_{l} j-\left(1+\sigma_{l}\right) / 2}\right) .
\end{aligned}
$$


The proof of Proposition 3.7, which is divided into the following four lemmas, consists essentially in manipulating each of the elements to obtain recurrence relations, and using induction to obtain expressions for $T_{k, r}, I_{k}, O_{k, l}$ and $J_{k, l}$ in $\overline{\left\langle\sigma^{2}\right\rangle}$. Part (c) of Proposition 3.7 is obtained by Abelianising these expressions.

Lemma 3.8. If $k \in \mathbb{Z}$ and $r \in\{0,1\}$ then $T_{k, r}=\prod_{i=1}^{\sigma_{k} k} B_{0, k-\sigma_{k} i-r+\left(\sigma_{k}+1\right) / 2}^{\sigma_{k}}$.

Proof. Let $r \in\{0,1\}$. Then clearly $T_{0, r}=\mathbf{1}$ and $T_{1, r}=u\left(B^{\varepsilon_{r}} u^{-\varepsilon_{r}}\right)^{\varepsilon_{r}}=B_{0,1-r}$, so the result holds for $k=1$. Suppose that the formula is valid for some $k \geq 1$. Then:

$$
\begin{aligned}
T_{k+1, r} & =u^{k+1}\left(B^{\varepsilon_{r}} u^{-\varepsilon_{r}}\right)^{(k+1) \varepsilon_{r}}=u \cdot u^{k}\left(B^{\varepsilon_{r}} u^{-\varepsilon_{r}}\right)^{k \varepsilon_{r}} \cdot\left(B^{\varepsilon_{r}} u^{-\varepsilon_{r}}\right)^{\varepsilon_{r}} \\
& =u T_{k, r} u^{-1} \cdot u\left(B^{\varepsilon_{r}} u^{-\varepsilon_{r}}\right)^{\varepsilon_{r}}=u\left(\prod_{i=1}^{k} B_{0, k-i+1-r}\right) u^{-1} \cdot B_{0,1-r}=\prod_{i=1}^{k+1} B_{0, k+1-i+1-r},
\end{aligned}
$$

and so the result holds for all $k \geq 0$ by induction. Suppose that $k \geq 1$. Then:

$$
\begin{aligned}
T_{-k, r} & =u^{-k}\left(B^{\varepsilon_{r}} u^{-\varepsilon_{r}}\right)^{-k \varepsilon_{r}}=u^{-k}\left(u^{k}\left(B^{\varepsilon_{r}} u^{-\varepsilon_{r}}\right)^{k \varepsilon_{r}}\right)^{-1} u^{k}=u^{-k} T_{k, r}^{-1} u^{k} \\
& =\left(\prod_{i=1}^{k} B_{0,-i+1-r}\right)^{k}=\prod_{i=1}^{k} B_{0,-k+i-r}^{-1}
\end{aligned}
$$

using the result for $T_{k, r}$ in the case $k \geq 1$, which proves the formula for all $k \in \mathbb{Z}$.

Lemma 3.9. Let $k \in \mathbb{Z}$. If $k=0$ then $I_{k}=\mathbf{1}$, and if $k \neq 0$ then:

$$
I_{k}=\left(\prod_{i=1}^{\sigma_{k} k} B_{i+k\left(1-\sigma_{k}\right) / 2,0}\right)^{-\sigma_{k}}
$$

Proof. If $k=0$, then clearly $I_{0}=\mathbf{1}$. If $k=1, I_{1}=v(v B)^{-1}=v B^{-1} v^{-1}=B_{1,0}^{-1}$, and result holds in this case. Suppose that the formula for $I_{k}$ holds for some $k \geq 1$, and let us prove the formula for $k+1$. We have:

$$
\begin{aligned}
I_{k+1} & =v^{k+1}(v B)^{-k-1}=v v^{k}(v B)^{-k} v^{-1} v(v B)^{-1}=v I_{k} v^{-1} I_{1} \\
& =v\left(\prod_{i=1}^{k} B_{i, 0}\right)^{-1} v^{-1} B_{1,0}^{-1}=\left(\prod_{i=1}^{k} B_{i+1,0}\right)^{-1} B_{1,0}^{-1}=\left(\prod_{i=1}^{k+1} B_{i, 0}\right)^{-1},
\end{aligned}
$$

so by induction, the formula for $I_{k}$ holds for all $k \geq 0$. If $k<0$ then $-k>0$ and so:

$$
\begin{aligned}
I_{k} & =v^{k}(v B)^{-k}=v^{k}(v B)^{-k} v^{k} v^{-k}=v^{k}\left(v^{-k}(v B)^{k}\right)^{-1} v^{-k}=v^{k} I_{-k}^{-1} v^{-k} \\
& =v^{k}\left(\prod_{i=1}^{-k} B_{i, 0}\right) v^{-k}=\prod_{i=1}^{-k} B_{k+i, 0} .
\end{aligned}
$$

It follows that the formula given in the statement holds for all $k \neq 0$.

Lemma 3.10. Let $k, l \in \mathbb{Z}$, let $\varepsilon \in\{-1,1\}$, and let $\omega=\left\{\begin{array}{ll}0 & \text { if } \varepsilon=-1 \\ 1 & \text { if } \varepsilon=1 .\end{array}\right.$ If $k=0$ or $l=0$, then $J_{k, l}=\mathbf{1}$, and if $k, l>0$, then:

$$
J_{k, \varepsilon l}=\prod_{i=1}^{k}\left(\prod_{j=1}^{l} B_{2 k-2 i+1, \varepsilon j-\omega}^{-\varepsilon}\right) \text { and } J_{-k, \varepsilon l}=\left(\prod_{i=1}^{k}\left(\prod_{j=1}^{l} B_{-2 i+1, \varepsilon j-\omega}^{-\varepsilon}\right)\right)^{-1} .
$$


Proof. If $k=0$ or $l=0$, then clearly $J_{k, l}=\mathbf{1}$. Now let $k=1$ and $\varepsilon \in\{1,-1\}$. Then for all $l \geq 0$, we have:

$$
\begin{aligned}
J_{1, \varepsilon(l+1)} & =v^{2}\left(v u^{\varepsilon(l+1)}\right)^{-2}=v^{2} u^{-\varepsilon} u^{-\varepsilon l} v^{-1} u^{-\varepsilon} u^{-\varepsilon l} v^{-1} \\
& =v^{2}\left(u^{-\varepsilon l} v^{-1} u^{-\varepsilon l} v^{-1}\right) v u^{\varepsilon l} v u^{-\varepsilon} v^{-1} u^{-\varepsilon} u^{-\varepsilon l} v^{-1} \\
& =J_{1, \varepsilon l} v u^{\varepsilon l+(\varepsilon-1) / 2}\left(u^{(1-\varepsilon) / 2} v u^{-\varepsilon} v^{-1} u^{-\varepsilon+(\varepsilon-1) / 2}\right) u^{-\varepsilon l+(1-\varepsilon) / 2} v^{-1} \\
& =J_{1, \varepsilon l} v u^{\varepsilon l+(\varepsilon-1) / 2} B^{-\varepsilon} u^{-\varepsilon l+(1-\varepsilon) / 2} v^{-1}=J_{1, \varepsilon l} B_{1, \varepsilon l+(\varepsilon-1) / 2}^{-\varepsilon}
\end{aligned}
$$

Hence $J_{1,1}=B_{1,0}^{-1}$ and $J_{1,-1}=B_{1,-1}$, which are in agreement with the expressions of the statement of the lemma for $k=l=1$. Suppose now that these expressions hold for $k=1$ and some $l \geq 1$. Now $\varepsilon l+(\varepsilon-1) / 2=\varepsilon(l+1)-(\varepsilon+1) / 2=\varepsilon(l+1)-\omega$, so by (18), we have:

$$
J_{1, \varepsilon(l+1)}=J_{k, \varepsilon l}=\left(\prod_{j=1}^{l} B_{1, \varepsilon j-\omega}^{-\varepsilon}\right) B_{1, \varepsilon l+(\varepsilon-1) / 2}^{-\varepsilon}=\prod_{j=1}^{l+1} B_{1, \varepsilon j-\omega}^{-\varepsilon},
$$

and thus the expressions of the statement hold for all $k, l \geq 1$ by induction. From this, for all $k, l>0$ and $\varepsilon \in\{1,-1\}$, it follows that:

$$
\begin{aligned}
J_{-k, \varepsilon l} & =v^{-2 k}\left(v u^{\varepsilon l}\right)^{2 k}=v^{-2 k}\left(v^{2 k}\left(v u^{\varepsilon l}\right)^{-2 k}\right)^{-1} v^{2 k}=v^{-2 k} J_{k, \varepsilon l}^{-1} v^{2 k} \\
& =v^{-2 k}\left(\prod_{i=1}^{k}\left(\prod_{j=1}^{l} B_{2 k-2 i+1, \varepsilon j-\omega}^{-\varepsilon}\right)\right)^{-1} v^{2 k}=\left(\prod_{i=1}^{k}\left(\prod_{j=1}^{l} B_{-2 i+1, \varepsilon j-\omega}^{-\varepsilon}\right)\right)^{-1},
\end{aligned}
$$

which completes the proof of the lemma.

Lemma 3.11. Let $k, l \in \mathbb{Z}$, let $\varepsilon \in\{-1,1\}$, and let $\omega=\left\{\begin{array}{ll}0 & \text { if } \varepsilon=-1 \\ 1 & \text { if } \varepsilon=1 .\end{array}\right.$ If $k=0$ or $l=0$, then $O_{k, l}=1$. If $k, l>0$, then for all $1 \leq i \leq k$ and $1 \leq j \leq l$, there exist $\eta_{i, j, \varepsilon}, \zeta_{i, j, \varepsilon}, \mu_{i, j, \varepsilon}, \nu_{i, j, \varepsilon} \in \overline{\left\langle\sigma^{2}\right\rangle}$ such that:

(a) $O_{\varepsilon k, l}=\prod_{j=1}^{l}\left(\prod_{i=1}^{k} \eta_{i, j, \varepsilon} B_{2 \omega k-2 i+1,-j} \eta_{i, j, \varepsilon}^{-1} \zeta_{i, j, \varepsilon} B_{2 \omega k-2 i, j-1}^{-1} \zeta_{i, j, \varepsilon}^{-1}\right)^{\varepsilon}$.

(b) $O_{\varepsilon k,-l}=\left(\prod_{j=1}^{l}\left(\prod_{i=1}^{k} \mu_{i, j, \varepsilon} B_{2 \omega k-2 i+1, l-j} \mu_{i, j, \varepsilon}^{-1} \nu_{i, j, \varepsilon} B_{2 \omega k-2 i,-l+j-1}^{-1} \nu_{i, j, \varepsilon}^{-1}\right)^{\varepsilon}\right)^{-1}$.

By taking $\varepsilon=1$ or -1 in parts (a) and (b) of Lemma 3.11 and Abelianising, one may check that the formula for $\widetilde{O}_{k, l}$ given in Proposition 3.7 is correct.

Proof of Lemma 3.11. If $k=0$ or $l=0$, then clearly $O_{k, l}=\mathbf{1}$. So suppose that $k, l>0$, and let $\varepsilon \in\{-1,1\}$. We start by considering the case $l=1$. If $k=\varepsilon=1$ then:

$$
O_{1,1}=v^{2} u v^{-2} u^{-1}=v u^{-1} u v u v^{-1} u v^{-1} v u^{-1} v^{-1} u^{-1}=v u^{-1} B u v^{-1} B^{-1}=B_{1,-1} B_{0,0}^{-1},
$$

and so the expression for $O_{1,1}$ given in (a) is correct by taking $\eta_{1,1,1}=\zeta_{1,1,1}=\mathbf{1}$. We now suppose that the expression for $O_{k, 1}$ given in (a) holds for some $k \geq 1$, where $\eta_{i, 1,1}=\zeta_{i, 1,1}=\mathbf{1}$ for all $1 \leq i \leq k$. Then:

$$
O_{k+1,1}=v^{2(k+1)} u v^{-2(k+1)} u^{-1}=v^{2} v^{2 k} u v^{-2 k} u^{-1} v^{-2} v^{2} u v^{-2} u^{-1}=v^{2} O_{k, 1} v^{-2} O_{1,1}
$$




$$
\begin{aligned}
& =v^{2}\left(\prod_{i=1}^{k} B_{2 k-2 i+1,-1} B_{2 k-2 i, 0}^{-1}\right) v^{-2} B_{1,-1} B_{0,0}^{-1} \\
& =\left(\prod_{i=1}^{k} B_{2(k+1)-2 i+1,-1} B_{2(k+1)-2 i, 0}^{-1}\right) B_{1,-1} B_{0,0}^{-1}=\prod_{i=1}^{k+1} B_{2(k+1)-2 i+1,-1} B_{2(k+1)-2 i, 0}^{-1} .
\end{aligned}
$$

By induction, it follows that that the expression for $O_{k, 1}$ given in (a) is correct for all $k \geq 1$, where $\eta_{i, 1,1}=\zeta_{i, 1,1}=1$ for all $1 \leq i \leq k$. Using this, for all $k \geq 1$, we obtain:

$$
\begin{aligned}
O_{-k, 1} & =v^{-2 k} u v^{2 k} u^{-1}=v^{-2 k}\left(v^{2 k} u v^{-2 k} u^{-1}\right)^{-1} v^{2 k}=v^{-2 k} O_{k, 1}^{-1} v^{2 k} \\
& =v^{-2 k}\left(\prod_{i=1}^{k} B_{2 k-2 i+1,-1} B_{2 k-2 i, 0}^{-1}\right)^{-1} v^{2 k}=\left(\prod_{i=1}^{k} B_{-2 i+1,-1} B_{-2 i, 0}^{-1}\right)^{-1} .
\end{aligned}
$$

Hence the expression for $O_{-k, 1}$ given in (a) is correct for all $k \geq 1$, where $\eta_{i, 1,-1}=\zeta_{i, 1,-1}=\mathbf{1}$ for all $1 \leq i \leq k$. We now suppose that the expression for $O_{\varepsilon k, l}$ given in (a) is correct for some $l \geq 1$ and all $k \geq 1$ and $\varepsilon \in\{1,-1\}$, where $\eta_{i, j, \varepsilon}, \zeta_{i, j, \varepsilon} \in \overline{\left\langle\sigma^{2}\right\rangle}$ for all $1 \leq i \leq k$ and $1 \leq j \leq l$. We just saw that this is the case if $l=1$. We now study the case $l+1$. By Lemma 3.6(c), for all $1 \leq i \leq k$, there exist $\eta_{i, l+1, \varepsilon}, \zeta_{i, l+1, \varepsilon} \in \overline{\left\langle\sigma^{2}\right\rangle}$ such that $u^{l} B_{2 \omega k-2 i+1,-1} u^{-l}=\eta_{i, l+1, \varepsilon} B_{2 \omega k-2 i+1,-l-1} \eta_{i, l+1, \varepsilon}^{-1}$ and $u^{l} B_{2 \omega k-2 i, 0} u^{-l}=\zeta_{i, l+1, \varepsilon} B_{2 \omega k-2 i, l} \zeta_{i, l+1, \varepsilon}^{-1}$. Thus:

$$
\begin{aligned}
O_{\varepsilon k, l+1}= & v^{2 \varepsilon k} u^{l+1} v^{-2 \varepsilon k} u^{-l-1}=v^{2 \varepsilon k} u^{l} v^{-2 \varepsilon k} u^{-l} u^{l} v^{2 \varepsilon k} u v^{-2 \varepsilon k} u^{-1} u^{-l} \\
= & O_{\varepsilon k, l} u^{l} O_{\varepsilon k, 1} u^{-l} \\
= & \prod_{j=1}^{l}\left(\prod_{i=1}^{k} \eta_{i, j, \varepsilon} B_{2 \omega k-2 i+1,-j} \eta_{i, j, \varepsilon}^{-1} \zeta_{i, j, \varepsilon} B_{2 \omega k-2 i, j-1}^{-1} \zeta_{i, j, \varepsilon}^{-1}\right)^{\varepsilon} \\
& u^{l}\left(\prod_{i=1}^{k} B_{2 \omega k-2 i+1,-1} B_{2 \omega k-2 i, 0}^{-1}\right)^{\varepsilon} u^{-l} \\
= & \prod_{j=1}^{l}\left(\prod_{i=1}^{k} \eta_{i, j, \varepsilon} B_{2 \omega k-2 i+1,-j} \eta_{i, j, \varepsilon}^{-1} \zeta_{i, j, \varepsilon} B_{2 \omega k-2 i, j-1}^{-1} \zeta_{i, j, \varepsilon}^{-1}\right)^{\varepsilon} . \\
= & \prod_{j=1}^{k}\left(\prod_{i=1}^{k} \eta_{i, l+1, \varepsilon} B_{2 \omega k-2 i+1,-l-1} \eta_{i, l+1, \varepsilon}^{-1} \zeta_{i, l+1, \varepsilon} B_{2 \omega k-2 i, l} \zeta_{i, l+1, \varepsilon}^{-1}\right)^{\varepsilon} \\
&
\end{aligned}
$$

By induction, it follows that (a) holds for all $k, l \geq 1$ and $\varepsilon \in\{1,-1\}$. We now use part (a) to prove part (b). Let $k, l \geq 1$ and $\varepsilon \in\{1,-1\}$. By Lemma 3.6(c), for all $1 \leq i \leq k$ and $1 \leq j \leq l$, there exist $\mu_{i, j, \varepsilon}, \nu_{i, j, \varepsilon} \in \overline{\left\langle\sigma^{2}\right\rangle}$ such that $u^{-l} \eta_{i, j, \varepsilon} B_{2 w k-2 i+1,-j} \eta_{i, j, \varepsilon}^{-1} u^{l}=\mu_{i, j, \varepsilon} B_{2 \omega k-2 i+1, l-j} \mu_{i, j, \varepsilon}^{-1}$ and $u^{-l} \zeta_{i, j, \varepsilon} B_{2 w k-2 i, j-1} \zeta_{i, j, \varepsilon}^{-1} u^{l}=\nu_{i, j, \varepsilon} B_{2 \omega k-2 i,-l+j-1} \nu_{i, j, \varepsilon}^{-1}$.

$$
\begin{aligned}
O_{\varepsilon k,-l} & =v^{2 \varepsilon k} u^{-l} v^{-2 \varepsilon k} u^{l}=u^{-l}\left(v^{2 \varepsilon k} u^{l} v^{-2 \varepsilon k} u^{-l}\right)^{-1} u^{l}=u^{-l} O_{2 \varepsilon k, l}^{-1} u^{l} \\
& =u^{-l}\left(\prod_{j=1}^{l}\left(\prod_{i=1}^{k} \eta_{i, j, \varepsilon} B_{2 \omega k-2 i+1,-j} \eta_{i, j, \varepsilon}^{-1} \zeta_{i, j, \varepsilon} B_{2 \omega k-2 i, j-1}^{-1} \zeta_{i, j, \varepsilon}^{-1}\right)^{\varepsilon}\right)^{-1} u^{l} \\
& =\left(\prod_{j=1}^{l}\left(\prod_{i=1}^{k} \mu_{i, j, \varepsilon} B_{2 \omega k-2 i+1, l-j} \mu_{i, j, \varepsilon}^{-1} \nu_{i, j, \varepsilon} B_{2 \omega k-2 i,-l+j-1}^{-1} \nu_{i, j, \varepsilon}^{-1}\right)^{\varepsilon}\right)^{-1}
\end{aligned}
$$

which proves part (b). 


\section{Proof of Theorem 1.3}

Let $\alpha \in\left[\mathbb{T}^{2}, * ; \mathbb{K}^{2}, *\right]$ and $\beta \in\left[\mathbb{T}^{2}, \mathbb{K}^{2}\right]$. With the notation of $[5$, Theorem 4$]$, suppose that $\alpha_{\mathcal{F}}=\beta$. According to [5, Theorem 7], the pointed homotopy class $\alpha$ has the Borsuk-Ulam property with respect to the free involution $\tau_{1}$ if and only if the homotopy class $\beta$ has the Borsuk-Ulam property with respect to $\tau_{1}$. So to obtain Theorem 1.3, it suffices to prove the statement for pointed homotopy classes. Before doing so, we give an algebraic criterion, similar to that of [5, Lemma 22], to decide whether a pointed homotopy class has the Borsuk-Ulam property with respect to $\tau_{1}$.

Lemma 4.1. Let $\alpha \in\left[\mathbb{T}^{2}, * ; \mathbb{K}^{2}, *\right]$ be a pointed homotopy class. Then $\alpha$ does not have the Borsuk-Ulam property with respect to $\tau_{1}$ if and only if there exist $a, b \in P_{2}\left(\mathbb{K}^{2}\right)$ such that:

(i) $a l_{\sigma}(b)=b a$.

(ii) $\left(p_{1}\right)_{\#}\left(a l_{\sigma}(a)\right)=\alpha_{\#}(1,0)$.

(iii) $\left(p_{1}\right)_{\#}(b)=\alpha_{\#}(0,1)$.

Proof. The result may be obtained in a manner similar to that of [5, Lemma 22], using Proposition 3.1 instead of [5, Theorem 12].

Corollary 4.2. Let $\alpha, \alpha^{\prime} \in\left[\mathbb{T}^{2}, * ; \mathbb{K}^{2}, *\right]$ be pointed homotopy classes, and suppose that:

$$
\alpha_{\#}:\left\{\begin{aligned}
(1,0) & \mapsto\left(r_{1}, s_{1}\right) \\
(0,1) & \mapsto\left(r_{2}, s_{2}\right)
\end{aligned} \quad \text { and } \alpha_{\#}^{\prime}:\left\{\begin{array}{l}
(1,0) \mapsto\left(r_{1}, s_{1}^{\prime}\right) \\
(0,1) \mapsto\left(r_{2}, s_{2}^{\prime}\right)
\end{array}\right.\right.
$$

for some $r_{1}, r_{2}, s_{1}, s_{1}^{\prime}, s_{2}, s_{2}^{\prime} \in \mathbb{Z}$. If $s_{1} \equiv s_{1}^{\prime} \bmod 4$ and $s_{2} \equiv s_{2}^{\prime} \bmod 2$ then $\alpha$ has the Borsuk-Ulam property with respect to $\tau_{1}$ if and only if $\alpha^{\prime}$ does.

Proof. Since the statement is symmetric with respect to $\alpha$ and $\alpha^{\prime}$, it suffices to show that if $\alpha$ does not have the Borsuk-Ulam property then neither does $\alpha^{\prime}$. If $\alpha$ does not have the Borsuk-Ulam property, there exist $a, b \in P_{2}\left(\mathbb{K}^{2}\right)$ satisfying (i)-(iii) of Lemma 4.1. By hypothesis, there exist $k_{1}, k_{2} \in \mathbb{Z}$ such that $s_{1}^{\prime}=s_{1}+4 k_{1}$ and $s_{2}^{\prime}=s_{2}+2 k_{2}$. Let $a^{\prime}=a\left(\mathbf{1} ; 0,2 k_{1}\right)$ and $b^{\prime}=b\left(\mathbf{1} ; 0,2 k_{2}\right)$ in $P_{2}\left(\mathbb{K}^{2}\right)$. It suffices to show that $a^{\prime}$ and $b^{\prime}$ satisfy (i)-(iii) of Lemma 4.1.Using Proposition 3.1, one may check that the centre of $B_{2}\left(\mathbb{K}^{2}\right)$ is the subgroup $\langle(\mathbf{1} ; 0,2)\rangle$. Thus:

$$
\begin{aligned}
a^{\prime} l_{\sigma}\left(b^{\prime}\right) & =a\left(\mathbf{1} ; 0,2 k_{1}\right) l_{\sigma}\left(b\left(\mathbf{1} ; 0,2 k_{2}\right)\right)=a l_{\sigma}(b)\left(\mathbf{1} ; 0,2 k_{1}+2 k_{2}\right) \stackrel{(\mathrm{i})}{=} b a\left(\mathbf{1} ; 0,2 k_{1}+2 k_{2}\right)=b^{\prime} a^{\prime}, \\
\left(p_{1}\right)_{\#}\left(a^{\prime} l_{\sigma}\left(a^{\prime}\right)\right) & =\left(p_{1}\right)_{\#}\left(a\left(\mathbf{1} ; 0,2 k_{1}\right) l_{\sigma}\left(a\left(\mathbf{1} ; 0,2 k_{1}\right)\right)\right)=\left(p_{1}\right)_{\#}\left(a l_{\sigma}(a)\left(\mathbf{1} ; 0,4 k_{1}\right)\right) \\
& \stackrel{(\mathrm{ii})}{=}\left(r_{1}, s_{1}\right)\left(0,4 k_{1}\right)=\left(r_{1}, s_{1}^{\prime}\right)=\alpha_{\#}^{\prime}(1,0), \text { and } \\
\left(p_{1}\right)_{\#}\left(b^{\prime}\right) & =\left(p_{1}\right)_{\#}\left(b\left(\mathbf{1} ; 0,2 k_{2}\right)\right) \stackrel{(\mathrm{iii})}{=}\left(r_{2}, s_{2}\right)\left(0,2 k_{2}\right)=\left(r_{2}, s_{2}^{\prime}\right)=\alpha_{\#}^{\prime}(0,1),
\end{aligned}
$$

which proves the corollary.

Remark 4.3. Let $\alpha \in\left[\mathbb{T}^{2}, * ; \mathbb{K}^{2}, *\right]$ be a pointed homotopy class, and let $\alpha_{\#}: \pi_{1}\left(\mathbb{T}^{2}\right) \rightarrow \pi_{1}\left(\mathbb{K}^{2}\right)$ be the homomorphism described in [5, Theorem 4], and that is of one of the four types given in Proposition 1.1.

(a) Suppose that $\alpha_{\#}$ is of Type 1,2 or 3 , and let $i \in\{0,1\}, s_{1}$ and $s_{2}$ be the integers that appear in the description of $\alpha_{\#}$ in Proposition 1.1. By Proposition 2.2 and Corollary 4.2, $\alpha$ has the Borsuk-Ulam property with respect to $\tau_{1}$ if and only if $\alpha^{\prime}$ does, where $\alpha^{\prime} \in\left[\mathbb{T}^{2}, * ; \mathbb{K}^{2}, *\right]$ satisfies: 
(i) $\alpha_{\#}^{\prime}(1,0)=\left(0,2 s_{1}+1 \bmod 4\right)$ and $\alpha_{\#}^{\prime}(0,1)=(0, j)$ if $\alpha_{\#}$ is of Type 1 (in which case $j=0$ ), or is of Type 2 (in which case $j=1$ ).

(ii) $\alpha_{\#}^{\prime}(1,0)=\left(0,2 s_{1} \bmod 4\right)$ and $\alpha_{\#}^{\prime}(0,1)=(0,1)$ if $\alpha_{\#}$ is of Type 3 .

So for each of Types 1, 2 and 3 , there are two cases to consider, $s_{1}=0$, and $s_{1}=1$.

(b) Suppose that $\alpha_{\#}$ is of Type 4, and let $r_{1}, r_{2}, s_{1}$ and $s_{2}$ be the integers that appear in the description of $\alpha_{\#}$ in Proposition 1.1, where $r_{1} \geq 0$. By Proposition 2.2 and Corollary 4.2, $\alpha$ has the Borsuk-Ulam property with respect to $\tau_{1}$ if and only if $\alpha^{\prime}$ does, where $\alpha^{\prime} \in\left[\mathbb{T}^{2}, * ; \mathbb{K}^{2}, *\right]$ satisfies $\alpha_{\#}^{\prime}(1,0)=\left(r_{1}, 2 s_{1} \bmod 4\right)$ and $\alpha_{\#}^{\prime}(0,1)=\left(r_{2}, 0\right)$. So for each pair of integers $\left(r_{1}, r_{2}\right)$, where $r_{1} \geq 0$, there are two cases to consider, $s_{1}=0$, and $s_{1}=1$.

To prove Theorem 1.3, it suffices to study the cases described by Remark 4.3. This will be carried out in Propositions 4.4-4.7 below. Part of Proposition 4.4 (resp. Proposition 4.5) treats the cases of Remark 4.3(a)(i) (resp. Remark 4.3(a)(ii)), and part of Proposition 4.4 and Propositions 4.6 and 4.7 deal with the cases of Remark 4.3(b). In each case, we will make use of Proposition 3.1 and its notation, as well as the commutative diagram (5).

Proposition 4.4. Let:

$$
\Sigma=\{(0,2 s+1,0, j) \mid j, s \in\{0,1\}\} \cup\left\{\left(r_{1}, 0, r_{2}, 0\right) \mid r_{1}, r_{2} \in \mathbb{Z}, r_{1} \geq 0\right\} \cup\{(0,2,0,0)\} .
$$

Let $\alpha \in\left[\mathbb{T}^{2}, * ; \mathbb{K}^{2}, *\right]$ be a pointed homotopy class such that $\alpha_{\#}:\left\{\begin{array}{l}(1,0) \mapsto(\rho, \gamma) \\ (0,1) \mapsto(\xi, \tau),\end{array}\right.$ where $(\rho, \gamma, \xi, \tau) \in \Sigma$. Then $\alpha$ does not have the Borsuk-Ulam property with respect to $\tau_{1}$.

Proposition 4.5. Let $\alpha \in\left[\mathbb{T}^{2}, * ; \mathbb{K}^{2}, *\right]$ be a pointed homotopy class such that $\alpha_{\#}:\left\{\begin{array}{l}(1,0) \mapsto(0,2 s) \\ (0,1) \mapsto(0,1),\end{array}\right.$ where $s \in\{0,1\}$. Then $\alpha$ has the Borsuk-Ulam property with respect to $\tau_{1}$.

Proposition 4.6. If $\alpha_{\#}:\left\{\begin{array}{l}(1,0) \mapsto\left(r_{1}, 2\right) \\ (0,1) \mapsto\left(r_{2}, 0\right),\end{array}\right.$ where $r_{1}, r_{2} \in \mathbb{Z}, r_{1}>0$, and one of the following conditions holds:

(a) $r_{2}=0$

(b) $r_{2} \neq 0$ and $e\left(r_{1}\right) \leq e\left(r_{2}\right)$.

Then $\alpha$ does not have the Borsuk-Ulam property with respect to $\tau_{1}$.

Proposition 4.7. Let $\alpha \in\left[\mathbb{T}^{2}, * ; \mathbb{K}^{2}, *\right]$ be a pointed homotopy class such that $\alpha_{\#}:\left\{\begin{array}{l}(1,0) \mapsto\left(r_{1}, 2\right) \\ (0,1) \mapsto\left(r_{2}, 0\right) \text {, }\end{array}\right.$ where $r_{1}, r_{2} \in \mathbb{Z}, r_{1} \geq 0, r_{2} \neq 0$, and one of the following conditions holds:

(a) $r_{1}=0$.

(b) $r_{1}>0$, and $e\left(r_{1}\right)>e\left(r_{2}\right)$.

Then $\alpha$ has the Borsuk-Ulam property with respect to $\tau_{1}$.

The following lemma will be used in the proofs of some of these propositions. 
Lemma 4.8. Let $a, b \in P_{2}\left(\mathbb{K}^{2}\right)$. Then there exist $x, y \in \overline{\left\langle\sigma^{2}\right\rangle}$ and $a_{i}, b_{i}, m_{i}, n_{i} \in \mathbb{Z}$, where $i \in\{1,2\}$, such that:

$$
a=\left(u^{a_{1}} v^{a_{2}} x ; m_{1}, n_{1}\right) \text { and } b=\left(u^{b_{1}} v^{b_{2}} y ; m_{2}, n_{2}\right) .
$$

Suppose further that $a$ and $b$ satisfy the relation of Lemma 4.1(i). Then:

$$
b_{2}=0 \text { and }\left(1+(-1)^{\delta_{n_{1}}+1}\right) m_{2}=\left(1+(-1)^{\delta_{n_{2}}+1}\right) m_{1}+(-1)^{\delta_{n_{1}}} b_{1},
$$

so $b_{1}$ is even, and:

$$
\begin{aligned}
& u^{b_{1}} y B^{m_{2}-\delta_{n_{2}}} u^{a_{1} \varepsilon_{n_{2}}}\left(B^{\delta_{n_{2}}} v u^{-2 m_{2}}\right)^{a_{2}} B^{\delta_{n_{2}}-m_{2}} \theta\left(m_{2}, \delta_{n_{2}}\right)(x)= \\
& u^{a_{1}} v^{a_{2}} x B^{m_{1}-\delta_{n_{1}}}\left(B^{\varepsilon_{n_{1}}} u^{-\varepsilon_{n_{1}}}\right)^{b_{1}} B^{-\varepsilon_{n_{1}} b_{1}+\delta_{n_{1}}-m_{1}} \theta\left(m_{1}+\varepsilon_{n_{1}} b_{1}, \delta_{n_{1}}\right)(\rho(y)) B^{\delta_{n_{2}} \varepsilon_{n_{1}}} .
\end{aligned}
$$

Proof. Let $a, b \in P_{2}\left(\mathbb{K}^{2}\right)$. Proposition 3.5 implies that there exist $x, y \in \overline{\left\langle\sigma^{2}\right\rangle}$ and $a_{i}, b_{i}, m_{i}, n_{i} \in \mathbb{Z}$, where $i \in\{1,2\}$, for which (19) holds. First, we have:

$$
\begin{aligned}
b a & =\left(u^{b_{1}} v^{b_{2}} y ; m_{2}, n_{2}\right)\left(u^{a_{1}} v^{a_{2}} x ; m_{1}, n_{1}\right) \\
& =\left(u^{b_{1}} v^{b_{2}} y \theta\left(m_{2}, \delta_{n_{2}}\right)\left(u^{a_{1}} v^{a_{2}} x\right) ; m_{2}+(-1)^{\delta_{n_{2}}} m_{1}, n_{2}+n_{1}\right) .
\end{aligned}
$$

Now:

$$
\begin{aligned}
l_{\sigma}(b)= & \left(\left(B u^{-1}\right)^{b_{1}} B^{-b_{1}} ; b_{1}, 0\right)\left((u v)^{-b_{2}}(u B)^{\delta_{b_{2}}} ; 0, b_{2}\right)(\rho(y) ; 0,0)\left(\mathbf{1} ; m_{2}, 0\right)\left(B^{\delta_{n_{2}}} ; 0, n_{2}\right) \\
= & \left(\left(B u^{-1}\right)^{b_{1}} B^{-b_{1}} ; b_{1}, 0\right)\left((u v)^{-b_{2}}(u B)^{\delta_{b_{2}}} ; 0, b_{2}\right)\left(\rho(y) B^{\delta_{n_{2}}} ; m_{2}, n_{2}\right) \\
= & \left(\left(B u^{-1}\right)^{b_{1}} B^{-b_{1}} \theta\left(b_{1}, 0\right)\left((u v)^{-b_{2}}(u B)^{\delta_{b_{2}}}\right) ; b_{1}, b_{2}\right)\left(\rho(y) B^{\delta_{n_{2}}} ; m_{2}, n_{2}\right) \\
= & \left(\left(B u^{-1}\right)^{b_{1}} B^{-b_{1}} \theta\left(b_{1}, 0\right)\left((u v)^{-b_{2}}(u B)^{\delta_{b_{2}}}\right) \theta\left(b_{1}, \delta_{b_{2}}\right)\left(\rho(y) B^{\delta_{n_{2}}}\right) ;\right. \\
& \left.b_{1}+(-1)^{\delta_{b_{2}}} m_{2}, b_{2}+n_{2}\right) .
\end{aligned}
$$

Thus:

$$
\begin{aligned}
\left(p_{1}\right)_{\#}\left(a l_{\sigma}(b)\right) & =\left(m_{1}, n_{1}\right)\left(b_{1}+(-1)^{\delta_{b_{2}}} m_{2}, b_{2}+n_{2}\right) \\
& =\left(m_{1}+(-1)^{\delta_{n_{1}}} b_{1}+(-1)^{\delta_{n_{1}}+\delta_{b_{2}}} m_{2}, n_{1}+b_{2}+n_{2}\right) .
\end{aligned}
$$

Suppose that $a$ and $b$ satisfy the relation of Lemma 4.1(i). It follows from (22) and (24) that $b_{2}=0$, and then that $m_{2}+(-1)^{\delta_{n_{2}}} m_{1}=m_{1}+(-1)^{\delta_{n_{1}}} b_{1}+(-1)^{\delta_{n_{1}}} m_{2}$, which yields (20). This implies that $b_{1}$ is even. We now expand and simplify the remaining parts of (22) and (23):

$$
\begin{aligned}
p_{F}(b a) & =u^{b_{1}} y \theta\left(m_{2}, \delta_{n_{2}}\right)\left(u^{a_{1}} v^{a_{2}} x\right) \\
& =u^{b_{1}} y B^{m_{2}-\delta_{n_{2}}} u^{a_{1} \varepsilon_{n_{2}}}\left(B^{\delta_{n_{2}}} v u^{-2 m_{2}}\right)^{a_{2}} B^{\delta_{n_{2}}-m_{2}} \theta\left(m_{2}, \delta_{n_{2}}\right)(x),
\end{aligned}
$$

and

$$
p_{F}\left(l_{\sigma}(b)\right)=\left(B u^{-1}\right)^{b_{1}} B^{-b_{1}} \theta\left(b_{1}, 0\right)\left(\rho(y) B^{\delta_{n_{2}}}\right)=\left(B u^{-1}\right)^{b_{1}} B^{-b_{1}} \theta\left(b_{1}, 0\right)(\rho(y)) B^{\delta_{n_{2}}} .
$$

Hence:

$$
\begin{aligned}
p_{F}\left(a l_{\sigma}(b)\right) & =u^{a_{1}} v^{a_{2}} x \theta\left(m_{1}, \delta_{n_{1}}\right)\left(\left(B u^{-1}\right)^{b_{1}} B^{-b_{1}} \theta\left(b_{1}, 0\right)(\rho(y)) B^{\delta_{n_{2}}}\right) \\
& =u^{a_{1}} v^{a_{2}} x B^{m_{1}-\delta_{n_{1}}}\left(B^{\varepsilon_{n_{1}}} u^{-\varepsilon_{n_{1}}}\right)^{b_{1}} B^{-\varepsilon_{n_{1}} b_{1}+\delta_{n_{1}}-m_{1}} \theta\left(m_{1}+\varepsilon_{n_{1}} b_{1}, \delta_{n_{1}}\right)(\rho(y)) B^{\delta_{n_{2}} \varepsilon_{n_{1}}} .
\end{aligned}
$$

Equation (21) then follows from the hypothesis, and equations (25) and (26). 
Proof of Proposition 4.4. Let $\alpha \in\left[\mathbb{T}^{2}, * ; \mathbb{K}^{2}, *\right]$ be a pointed homotopy class such that $\alpha_{\#}(1,0)=$ $(\rho, \gamma)$ and $\alpha_{\#}(0,1)=(\xi, \tau)$, where $(\rho, \gamma, \xi, \tau) \in \Sigma$. Note that either $\rho=\xi=0$ or $\gamma=\tau=0$. In what follows, we will use the identities $\delta_{\delta_{q}}=\delta_{q}$ and $\varepsilon_{\delta_{q}}=\varepsilon_{q}$ for all $q \in \mathbb{Z}$. Let $a, b \in P_{2}\left(\mathbb{K}^{2}\right)$ be such that $a=\left(u^{\delta_{\rho}} v^{\delta_{\gamma}} B^{\delta_{\gamma}\left(\gamma-\delta_{\gamma}\right) / 2} ;\left(\rho-\delta_{\rho}\right) / 2,\left(\gamma-\delta_{\gamma}\right) / 2\right)$ and $b=\left(B^{-\delta_{\rho} \xi} ; \xi, \tau\right)$. With respect to the notation of (19), $x=B^{\delta_{\gamma}\left(\gamma-\delta_{\gamma}\right) / 2}$ and $y=B^{-\delta_{\rho} \xi}$. To prove the result, we show that conditions (i)(iii) of Lemma 4.1 are satisfied. Clearly, $\left(p_{1}\right)_{\#}(b)=(\xi, \tau)=\alpha_{\#}(0,1)$. Further, by taking $b=a$ in (24), we have:

$$
\left(p_{1}\right)_{\#}\left(a l_{\sigma}(a)\right)=\left(\left(1+(-1)^{\delta_{\left(\gamma-\delta_{\gamma}\right) / 2}+\delta_{\gamma}}\right) \frac{\left(\rho-\delta_{\rho}\right)}{2}+\varepsilon_{\left(\gamma-\delta_{\gamma}\right) / 2} \delta_{\rho}, \gamma\right) .
$$

If $\rho=\xi=0$ then the first coordinate of the right-hand side of (27) is equal to zero, while if $\gamma=\tau=$ 0 , this coordinate is equal to $\rho$. In both cases, we conclude that $\left(p_{1}\right)_{\#}\left(a l_{\sigma}(a)\right)=(\rho, \gamma)=\alpha_{\#}(1,0)$. Hence conditions (ii) and (iii) of Lemma 4.1 are satisfied. It remains to check condition (i). Note that in the proof of Lemma 4.8, the only condition that we have applied to obtain equations (25) and (26) is that $b_{2}=0$. But this coefficient is zero in our case, and so these equations are also satisfied here. So using (22), (25) and Proposition 3.1, we see that:

$$
\begin{aligned}
b a & =\left(B^{-\delta_{\rho} \xi} \theta\left(\xi, \delta_{\tau}\right)\left(u^{\delta_{\rho}} v^{\delta_{\gamma}} B^{\delta_{\gamma}\left(\gamma-\delta_{\gamma}\right) / 2}\right) ; \xi+\varepsilon_{\tau} \frac{\left(\rho-\delta_{\rho}\right)}{2}, \tau+\frac{\left(\gamma-\delta_{\gamma}\right)}{2}\right) \\
& =\left(B^{-\delta_{\rho} \xi} B^{\xi-\delta_{\tau}} u^{\delta_{\rho} \varepsilon_{\tau}}\left(B^{\delta_{\tau}} v u^{-2 \xi}\right)^{\delta_{\gamma}} B^{\delta_{\tau}-\xi} B^{\varepsilon_{\tau} \delta_{\gamma}\left(\gamma-\delta_{\gamma}\right) / 2} ; \xi+\varepsilon_{\tau} \frac{\left(\rho-\delta_{\rho}\right)}{2}, \tau+\frac{\left(\gamma-\delta_{\gamma}\right)}{2}\right) .
\end{aligned}
$$

In a similar manner, by (24) and (26), we see that:

$$
a l_{\sigma}(b)=\left(u^{\delta_{\rho}} v^{\delta_{\gamma}} B^{\delta_{\gamma}\left(\gamma-\delta_{\gamma}\right) / 2+\left(\delta_{\tau}-\delta_{\rho} \xi\right) \varepsilon_{\left(\gamma-\delta_{\gamma}\right) / 2}} ; \frac{\left(\rho-\delta_{\rho}\right)}{2}+\varepsilon_{\left(\gamma-\delta_{\gamma}\right) / 2} \xi, \tau+\frac{\left(\gamma-\delta_{\gamma}\right)}{2}\right) .
$$

If $\rho=\xi=0$ (resp. $\gamma=\tau=0)$ then $\left(p_{1}\right)_{\#}(b a)=\left(0, \tau+\left(\gamma-\delta_{\gamma}\right) / 2\right)=\left(p_{1}\right)_{\#}\left(a l_{\sigma}(b)\right)$ (resp. $\left.\left(p_{1}\right)_{\#}(b a)=\left(\xi+\left(\rho-\delta_{\rho}\right) / 2,0\right)=\left(p_{1}\right)_{\#}\left(a l_{\sigma}(b)\right)\right)$. So it remains to show that $p_{F}(b a)=p_{F}\left(a l_{\sigma}(b)\right)$.

(a) If $\rho=\xi=0, p_{F}(b a)=B^{-\delta_{\tau}}\left(B^{\delta_{\tau}} v\right)^{\delta_{\gamma}} B^{\delta_{\tau}} B^{\varepsilon_{\tau} \delta_{\gamma}\left(\gamma-\delta_{\gamma}\right) / 2}$ and $p_{F}\left(a l_{\sigma}(b)\right)=v^{\delta_{\gamma}} B^{\delta_{\gamma}\left(\gamma-\delta_{\gamma}\right) / 2+\delta_{\tau} \varepsilon_{\left(\gamma-\delta_{\gamma}\right) / 2}}$.

(i) Suppose that $\delta_{\gamma}=0$. Since $(\rho, \gamma, \xi, \tau) \in \Sigma$, it follows that $\delta_{\tau}=0$, and thus $p_{F}(b a)=$ $p_{F}\left(a l_{\sigma}(b)\right)=\mathbf{1}$.

(ii) Suppose that $\delta_{\gamma}=1$. Then $p_{F}(b a)=v B^{\delta_{\tau}+\varepsilon_{\tau}(\gamma-1) / 2}$ and $p_{F}\left(a l_{\sigma}(b)\right)=v B^{(\gamma-1) / 2+\delta_{\tau} \varepsilon_{(\gamma-1) / 2}}$. If $\delta_{\tau}=0$ then $p_{F}(b a)=v B^{(\gamma-1) / 2}=p_{F}\left(a l_{\sigma}(b)\right)$. So suppose that $\delta_{\tau}=1$. Since $(\rho, \gamma, \xi, \tau) \in \Sigma$, it follows that $(\gamma-1) / 2 \in\{0,1\}$, and one can check easily that $1-(\gamma-$ $1) / 2=(\gamma-1) / 2+\varepsilon_{(\gamma-1) / 2}$. It follows that $p_{F}(b a)=p_{F}\left(a l_{\sigma}(b)\right)$.

(b) If $\gamma=\tau=0$ then $p_{F}(b a)=B^{\left(1-\delta_{\rho}\right) \xi} u^{\delta_{\rho}} B^{-\xi}$ and $p_{F}\left(a l_{\sigma}(b)\right)=u^{\delta_{\rho}} B^{-\delta_{\rho} \xi}$, and one sees that $p_{F}(b a)=\mathbf{1}=p_{F}\left(a l_{\sigma}(b)\right)$ if $\delta_{\rho}=0$, and $p_{F}(b a)=u B^{-\xi}=p_{F}\left(a l_{\sigma}(b)\right)$ if $\delta_{\rho}=1$.

In order to prove Proposition 4.5, we will make use of the following lemma.

Lemma 4.9. Let $\alpha \in\left[\mathbb{T}^{2}, * ; \mathbb{K}^{2}, *\right]$ be such that $\alpha_{\#}:\left\{\begin{array}{l}(1,0) \mapsto(0,2 s) \\ (0,1) \mapsto(0,1)\end{array}\right.$ for some $s \in\{0,1\}$. If $\alpha$ does not have the Borsuk-Ulam property with respect to $\tau_{1}$ then there exist $x, y \in{\overline{\left\langle\sigma^{2}\right\rangle}}_{A b}$ and $m_{1}, n_{1} \in \mathbb{Z}$ that satisfy the following equation in ${\overline{\left\langle\sigma^{2}\right\rangle}}_{A b}$ :

$$
\mu_{1}(x)+\mu_{2}(y)=\widetilde{I}_{2 n_{1}-2 s}-\widetilde{T}_{-2 m_{1}, \delta_{n_{1}}}-\widetilde{O}_{n_{1}-s,-2 m_{1}}-\left(m_{1}+\delta_{n_{1}}+\varepsilon_{n_{1}}\right) B_{0,0}-\left(m_{1}-\delta_{n_{1}}\right) B_{0,-2 m_{1}}
$$




$$
-B_{2 n_{1}-2 s,-2 \delta_{n_{1}+1} m_{1}}+B_{2 n_{1}-2 s, 0}
$$

where $\mu_{1}, \mu_{2}:{\overline{\left\langle\sigma^{2}\right\rangle}}_{A b} \rightarrow{\overline{\left\langle\sigma^{2}\right\rangle}}_{A b}$ are the homomorphisms defined on the basis elements of ${\overline{\left\langle\sigma^{2}\right\rangle}}_{A b}$ by:

$$
\begin{aligned}
& \mu_{1}\left(B_{k, l}\right)=B_{k, l-2 \varepsilon_{k} m_{1}}+B_{k,-l} \text { and } \\
& \mu_{2}\left(B_{k, l}\right)=\varepsilon_{k} \varepsilon_{\delta_{n_{1}}} B_{-k, \varepsilon_{\delta_{1}}} \varepsilon_{k+1} l+2 \delta_{k} m_{1}-B_{k+2 n_{1}-2 s, l-2 \varepsilon_{k} \delta_{n_{1}+1} m_{1} .} .
\end{aligned}
$$

Proof. By hypothesis, there exist $a, b \in P_{2}\left(\mathbb{K}^{2}\right)$ satisfying (i)-(iii) of Lemma 4.1. Proposition 3.5 implies that $a$ and $b$ may be written in the form (19). Lemma 4.1(iii) implies that $\left(p_{1}\right)_{\#}(b)=(0,1)$, so $\left(m_{2}, n_{2}\right)=(0,1)$. Using Lemma 4.1(ii) and taking $b=a$ in (23), we obtain:

$$
\begin{aligned}
(0,2 s) & =\left(p_{1}\right)_{\#}\left(a l_{\sigma}(a)\right)=\left(m_{1}, n_{1}\right)\left(a_{1}+(-1)^{a_{2}} m_{1}, n_{1}+a_{2}\right) \\
& =\left(m_{1}+(-1)^{n_{1}} a_{1}+(-1)^{n_{1}+a_{2}} m_{1}, 2 n_{1}+a_{2}\right) .
\end{aligned}
$$

It follows that $a_{2}=2 s-2 n_{1}$ is even and $a_{1}=-2 \delta_{n_{1}+1} m_{1}$. Lemmas 4.1(i) and 4.8 then imply that $b_{2}=0$ and $b_{1}=-2 \varepsilon_{n_{1}} m_{1}$. We now analyse (21), which holds because Lemma 4.1(i) does. The left-hand side of (21) is equal to $p_{F}(b a)$, and may be rewritten as:

$$
\begin{aligned}
p_{F}(b a)= & u^{b_{1}} y B^{-1} u^{-a_{1}}(B v)^{2 s-2 n_{1}} B \theta(0,1)(x)=u^{b_{1}} y B^{-1} u^{-a_{1}} B(v B)^{2 s-2 n_{1}} \theta(0,1)(x) \\
= & u^{b_{1}-a_{1}} v^{2 s-2 n_{1}} \cdot v^{2 n_{1}-2 s} u^{a_{1}} y u^{-a_{1}} v^{2 s-2 n_{1}} \cdot v^{2 n_{1}-2 s} u^{a_{1}} B^{-1} u^{-a_{1}} v^{2 s-2 n_{1}} . \\
& v^{2 n_{1}-2 s} B v^{2 s-2 n_{1}} \cdot v^{2 n_{1}-2 s}(v B)^{2 s-2 n_{1}} \theta(0,1)(x) \\
= & u^{b_{1}-a_{1}} v^{2 s-2 n_{1}} c_{2 n_{1}-2 s, a_{1}}(y) B_{2 n_{1}-2 s, a_{1}}^{-1} B_{2 n_{1}-2 s, 0} I_{2 n_{1}-2 s} \theta(0,1)(x),
\end{aligned}
$$

and the right-hand side of $(21)$ is equal to $p_{F}\left(a l_{\sigma}(b)\right)$, and may be rewritten as:

$$
\begin{aligned}
p_{F}\left(a l_{\sigma}(b)\right)= & u^{a_{1}} v^{2 s-2 n_{1}} x B^{m_{1}-\delta_{n_{1}}}\left(B^{\varepsilon_{n_{1}}} u^{-\varepsilon_{n_{1}}}\right)^{b_{1}} B^{m_{1}+\delta_{n_{1}}} \theta\left(-m_{1}, \delta_{n_{1}}\right)(\rho(y)) B^{\varepsilon_{n_{1}}} \\
= & u^{2 m_{1}+a_{1}} v^{2 s-2 n_{1}}\left[v^{2 n_{1}-2 s}, u^{-2 m_{1}}\right] c_{0,-2 m_{1}}(x) B_{0,-2 m_{1}}^{m_{1}-\delta_{n_{1}}} . \\
& u^{-2 m_{1}}\left(B^{\varepsilon_{n_{1}}} u^{-\varepsilon_{n_{1}}}\right)^{-2 \varepsilon_{n_{1}} m_{1}} B_{0,0}^{m_{1}+\delta_{n_{1}}} \theta\left(-m_{1}, \delta_{n_{1}}\right)(\rho(y)) B_{0,0}^{\varepsilon_{n_{1}}} \\
= & u^{2 m_{1}+a_{1}} v^{2 s-2 n_{1}} O_{n_{1}-s,-2 m_{1}} c_{0,-2 m_{1}}(x) B_{0,-2 m_{1}}^{m_{1}-\delta_{n_{1}}} T_{-2 m_{1}, \delta_{n_{1}}} B_{0,0}^{m_{1}+\delta_{n_{1}}} . \\
& \theta\left(-m_{1}, \delta_{n_{1}}\right)(\rho(y)) B_{0,0}^{\varepsilon_{n_{1}}},
\end{aligned}
$$

Since $p_{F}(b a)=p_{F}\left(a l_{\sigma}(b)\right)$, and using the fact that $b_{1}-a_{1}=2 m_{1}+a_{1}$, it follows by Abelianising (31) and (32) that:

$$
\begin{aligned}
& \left(c_{0,-2 m_{1}}\right)_{\mathrm{Ab}}(x)-\theta(0,1)_{\mathrm{Ab}}(x)+\theta\left(-m_{1}, \delta_{n_{1}}\right)_{\mathrm{Ab}} \circ \rho_{\mathrm{Ab}}(y)-\left(c_{2 n_{1}-2 s, a_{1}}\right)_{\mathrm{Ab}}(y)= \\
& \widetilde{I}_{2 n_{1}-2 s}-\widetilde{T}_{-2 m_{1}, \delta_{n_{1}}}-\widetilde{O}_{n_{1}-s,-2 m_{1}}-\left(m_{1}+\delta_{n_{1}}+\varepsilon_{n_{1}}\right) B_{0,0}-\left(m_{1}-\delta_{n_{1}}\right) B_{0,-2 m_{1}}-B_{2 n_{1}-2 s, a_{1}}+B_{2 n_{1}-2 s, 0}
\end{aligned}
$$

in ${\overline{\left\langle\sigma^{2}\right\rangle}}_{\mathrm{Ab}}$, where the projection of $x$ (resp. $y$ ) in ${\overline{\left\langle\sigma^{2}\right\rangle}}_{\mathrm{Ab}}$ is also denoted by $x$ (resp. $y$ ). By (15)-(17), for all $k, l \in \mathbb{Z}$, we have:

$$
\left(c_{0,-2 m_{1}}\right)_{\mathrm{Ab}}\left(B_{k, l}\right)-\theta(0,1)_{\mathrm{Ab}}\left(B_{k, l}\right)=B_{k, l-2 \varepsilon_{k} m_{1}}+B_{k,-l}=\mu_{1}\left(B_{k, l}\right)
$$

and

$$
\begin{aligned}
\theta\left(-m_{1}, \delta_{n_{1}}\right)_{\mathrm{Ab}} \circ \rho_{\mathrm{Ab}}\left(B_{k, l}\right)-\left(c_{2 n_{1}-2 s, a_{1}}\right)_{\mathrm{Ab}}\left(B_{k, l}\right) & =\theta\left(-m_{1}, \delta_{n_{1}}\right)_{\mathrm{Ab}}\left(\varepsilon_{k} B_{-k, \varepsilon_{k+1} l}\right)-B_{k+2 n_{1}-2 s, l+\varepsilon_{k} a_{1}} \\
& =\varepsilon_{k} \varepsilon_{\delta_{n_{1}}} B_{-k, \varepsilon_{\delta_{1}}} \varepsilon_{k+1} l+2 \delta_{k} m_{1}-B_{k+2 n_{1}-2 s, l+\varepsilon_{k} a_{1}} \\
& =\mu_{2}\left(B_{k, l}\right) .
\end{aligned}
$$

The result follows by noting that $a_{1}=-2 \delta_{n_{1}+1} m_{1}$. 
We are now able to complete the proof of Proposition 4.5.

Proof of Proposition 4.5. We argue by contradiction. Suppose that $\alpha$ does not have the BorsukUlam property with respect to $\tau_{1}$. Then there exist $x, y \in{\overline{\left\langle\sigma^{2}\right\rangle}}_{\mathrm{Ab}}$ that satisfy equation (30) given in the statement of Lemma 4.9. Let $\xi:{\overline{\left\langle\sigma^{2}\right\rangle}}_{\mathrm{Ab}} \rightarrow \mathbb{Z}_{2}$ be the homomorphism defined on the basis $\left\{B_{k, l}\right\}_{k, l \in \mathbb{Z}}$ of ${\overline{\left\langle\sigma^{2}\right\rangle}}_{\mathrm{Ab}}$ by $\xi\left(B_{k, l}\right)=\overline{1}$ for all $k, l \in \mathbb{Z}$. From the definition of the maps $\mu_{1}$ and $\mu_{2}$, it follows that the left-hand side is sent to $\overline{0}$. By Proposition 3.7, $\xi\left(\widetilde{I}_{2 n_{1}-2 s}\right)=\xi\left(\widetilde{T}_{-2 m_{1}, \delta_{n_{1}}}\right)=$ $\xi\left(\widetilde{O}_{n_{1}-s,-2 m_{1}}\right)=\overline{0}$, and it follows that the right-hand side is sent to $\bar{\varepsilon}_{n_{1}}$, which is different from $\overline{0}$. This yields a contradiction. We conclude that $\alpha$ has the Borsuk-Ulam property with respect to $\tau_{1}$.

Proof of Proposition 4.6. Let $\alpha \in\left[\mathbb{T}^{2}, * ; \mathbb{K}^{2}, *\right]$ be a pointed homotopy class such that $\alpha_{\#}(1,0)=$ $\left(r_{1}, 2\right)$ and $\alpha_{\#}(0,1)=\left(r_{2}, 0\right)$, where $r_{1}, r_{2} \in \mathbb{Z}$ are such that $r_{1}>0$, and either $r_{2}=0$, or $r_{2} \neq 0$ and $e\left(r_{1}\right) \leq e\left(r_{2}\right)$. Let $o\left(r_{1}\right)=r_{1} / 2^{e\left(r_{1}\right)}$, and let $m=r_{2} / 2^{e\left(r_{1}\right)}$. Then $o\left(r_{1}\right)>0$ is odd, and $m \in \mathbb{Z}$ by hypothesis. Let $\alpha^{\prime} \in\left[\mathbb{T}^{2}, * ; \mathbb{K}^{2}, *\right]$ be the homotopy class for which $\alpha_{\#}^{\prime}(1,0)=\left(r_{1}, 2 o\left(r_{1}\right)\right)$ and $\alpha_{\#}^{\prime}(0,1)=\left(r_{2}, 2 m\right)$. By Corollary 4.2, to prove the result, it suffices to exhibit $a, b \in P_{2}\left(\mathbb{K}^{2}\right)$ that satisfy conditions (i)-(iii) of Lemma 4.1 for $\alpha^{\prime}$. Let $c=$ $\left(u^{2^{e\left(r_{1}\right)}} v^{2} ; 0,0\right) \in P_{2}\left(\mathbb{K}^{2}\right)$, and let $a=(c \sigma)^{o\left(r_{1}\right)} \sigma^{-1}$ and $b=(c \sigma)^{2 m}$. Then $a, b \in P_{2}\left(\mathbb{K}^{2}\right)$, and by Proposition 3.1, we see that $\left(p_{1}\right)_{\#}\left(l_{\sigma}(c)\right)=\left(2^{e\left(r_{1}\right)}, 2\right)$. Now $a l_{\sigma}(b)=(c \sigma)^{o\left(r_{1}\right)} \sigma^{-1} \sigma(c \sigma)^{2 m} \sigma^{-1}=$ $(c \sigma)^{2 m}(c \sigma)^{o\left(r_{1}\right)} \sigma^{-1}=b a$, so condition (i) of Lemma 4.1 is satisfied. Next, $a=\left((c \sigma)^{2}\right)^{\left(o\left(r_{1}\right)-1\right) / 2} c=$ $\left(c l_{\sigma}(c) \sigma^{2}\right)^{\left(o\left(r_{1}\right)-1\right) / 2} c$, hence $l_{\sigma}(a)=\left(l_{\sigma}(c) \sigma^{2} c\right)^{\left(o\left(r_{1}\right)-1\right) / 2} l_{\sigma}(c)$, and since $\left(p_{1}\right)_{\#}(c)=\left(p_{1}\right)_{\#}\left(\sigma^{2}\right)=$ $(0,0)$, it follows that $\left(p_{1}\right)_{\#}\left(a l_{\sigma}(a)\right)=\left(\left(p_{1}\right)_{\#}\left(l_{\sigma}(c)\right)\right)^{o\left(r_{1}\right)}=\left(2^{e\left(r_{1}\right)}, 2\right)^{o\left(r_{1}\right)}=\left(2^{e\left(r_{1}\right)} o\left(r_{1}\right), 2 o\left(r_{1}\right)\right)=$ $\left(r_{1}, 2 o\left(r_{1}\right)\right)=\alpha_{\#}(1,0)$. So condition (ii) of Lemma 4.1 holds. Finally, $b=(c \sigma c \sigma)^{m}=\left(c l_{\sigma}(c) \sigma^{2}\right)^{m}$, so $\left(p_{1}\right)_{\#}(b)=\left(p_{1}\right)_{\#}\left(l_{\sigma}(c)\right)^{m}=\left(2^{e\left(r_{1}\right)}, 2\right)^{m}=\left(2^{e\left(r_{1}\right)} m, 2 m\right)=\left(r_{2}, 2 m\right)=\alpha_{\#}^{\prime}(0,1)$, and condition (iii) of Lemma 4.1 is satisfied, which proves the proposition.

The rest of this section is devoted to proving Proposition 4.7.

Lemma 4.10. Let $\alpha \in\left[\mathbb{T}^{2}, * ; \mathbb{K}^{2}, *\right]$ be a homotopy class such that $\alpha_{\#}: \pi_{1}\left(\mathbb{T}^{2}\right) \rightarrow \pi_{1}\left(\mathbb{K}^{2}\right)$ satisfies $\alpha_{\#}(1,0)=\left(r_{1}, 2\right)$ and $\alpha_{\#}(0,1)=\left(r_{2}, 0\right)$, where $r_{1}, r_{2} \in \mathbb{Z}$. With the notation of Proposition 3.7, if $\alpha$ does not have the Borsuk-Ulam property, then there exist $x, y \in{\overline{\left\langle\sigma^{2}\right\rangle}}_{A b}$ and $\left(m_{1}, n_{1}\right) \in \mathbb{Z} \rtimes \mathbb{Z}$ such that:

$$
\begin{aligned}
\mu(x)+\nu(y)= & \widetilde{J}_{n_{1}-1,-2 r_{2}}-\widetilde{O}_{n_{1}-1,2 \delta_{n_{1}} r_{2}}-\widetilde{T}_{2 \delta_{n_{1}} r_{2}, \delta_{n_{1}}}+ \\
& r_{2} B_{2\left(n_{1}-1\right), 2 \delta_{n_{1}+1} m_{1}-\varepsilon_{n_{1}} r_{1}}-\left(m_{1}-\delta_{n_{1}}\right) B_{0,2 \delta_{n_{1}} r_{2}}-\left(\delta_{n_{1}}\left(1-2 r_{2}\right)-m_{1}+r_{2}\right) B_{0,0}
\end{aligned}
$$

in ${\overline{\left\langle\sigma^{2}\right\rangle}}_{A b}$, where $\mu, \nu:{\overline{\left\langle\sigma^{2}\right\rangle}}_{A b} \rightarrow{\overline{\left\langle\sigma^{2}\right\rangle}}_{A b}$ are the homomorphisms defined on the elements of the basis $\left\{B_{k, l}\right\}_{k, l \in \mathbb{Z}}$ of ${\overline{\left\langle\sigma^{2}\right\rangle}}_{A b}$ by:

$$
\begin{aligned}
& \mu\left(B_{k, l}\right)=B_{k, l+2 \varepsilon_{k} \delta_{n_{1}} r_{2}}-B_{k, l-2 \delta_{k} r_{2}} \\
& \nu\left(B_{k, l}\right)=\varepsilon_{k} \varepsilon_{n_{1}} B_{-k, \varepsilon_{n_{1}} \varepsilon_{k+1} l-2 \delta_{k}\left(m_{1}+2 \delta_{n_{1}} r_{2}\right)}-B_{k+2\left(n_{1}-1\right), l+\varepsilon_{k}\left(2 \delta_{n_{1}+1} m_{1}-\varepsilon_{n_{1}} r_{1}\right)} .
\end{aligned}
$$

Proof. Let $\alpha \in\left[\mathbb{T}^{2}, * ; \mathbb{K}^{2}, *\right]$ be a homotopy class that does not have the Borsuk-Ulam property, and that is represented by the homomorphism $\alpha_{\#}: \pi_{1}\left(\mathbb{T}^{2}\right) \rightarrow \pi_{1}\left(\mathbb{K}^{2}\right)$ given by $\alpha_{\#}(1,0)=\left(r_{1}, 2\right)$ and $\alpha_{\#}(0,1)=\left(r_{2}, 0\right)$, where $r_{1}, r_{2} \in \mathbb{Z}$. Then there exist $a, b \in P_{2}\left(\mathbb{K}^{2}\right)$ satisfying conditions (i)(iii) of Lemma 4.1. We write these elements in the form of equation (19). Condition (iii) implies that $\left(m_{2}, n_{2}\right)=\left(r_{2}, 0\right)$, and taking $b=a$ in (24), condition (ii) implies that $\left(r_{1}, 2\right)=\left(m_{1}(1+\right.$ $\left.\left.(-1)^{n_{1}+a_{2}}\right)+(-1)^{n_{1}} a_{1}, 2 n_{1}+a_{2}\right)$. It follows that $a_{2}=2\left(1-n_{1}\right)$ and that $a_{1}=\varepsilon_{n_{1}} r_{1}-2 \delta_{n_{1}+1} m_{1}$. By $(20)$, we have $b_{2}=0$ and $\left(1+(-1)^{\delta_{n_{1}}+1}\right) m_{2}=\left(1+(-1)^{\delta_{n_{2}}+1}\right) m_{1}+(-1)^{\delta_{n_{1}}} b_{1}$, from which we 
see that $b_{1}=-2 \delta_{n_{1}} r_{2}$ using the fact that $n_{2}=0$. Substituting this information into (25) and (26), and using that fact that $\varepsilon_{n_{1}} \delta_{n_{1}}=-\delta_{n_{1}}$, we obtain:

$$
\begin{aligned}
& p_{F}(b a)=u^{-2 \delta_{n_{1}} r_{2}} y B^{r_{2}} u^{\varepsilon_{n_{1}} r_{1}-2 \delta_{n_{1}+1} m_{1}}\left(v u^{-2 r_{2}}\right)^{2\left(1-n_{1}\right)} B^{-r_{2}} \theta\left(r_{2}, 0\right)(x) \\
& =u^{\varepsilon_{n_{1}} r_{1}-2 \delta_{n_{1}} r_{2}-2 \delta_{n_{1}+1} m_{1}} v^{2\left(1-n_{1}\right)} \cdot v^{2\left(n_{1}-1\right)} u^{2 \delta_{n_{1}+1} m_{1}-\varepsilon_{n_{1}} r_{1}} y B^{r_{2}} . \\
& u^{\varepsilon_{n_{1}} r_{1}-2 \delta_{n_{1}+1} m_{1}} v^{2\left(1-n_{1}\right)} \cdot v^{2\left(n_{1}-1\right)}\left(v u^{-2 r_{2}}\right)^{2\left(1-n_{1}\right)} B_{0,0}^{-r_{2}} \theta\left(r_{2}, 0\right)(x) \\
& =u^{\varepsilon_{n_{1}} r_{1}-2 \delta_{n_{1}} r_{2}-2 \delta_{n_{1}+1} m_{1}} v^{2\left(1-n_{1}\right)} c_{2\left(n_{1}-1\right), 2 \delta_{n_{1}+1} m_{1}-\varepsilon_{n_{1}} r_{1}}(y) \text {. } \\
& B_{2\left(n_{1}-1\right), 2 \delta_{n_{1}+1} m_{1}-\varepsilon_{n_{1}} r_{1}}^{r_{2}} J_{n_{1}-1,-2 r_{2}} B_{0,0}^{-r_{2}} \theta\left(r_{2}, 0\right)(x), \\
& p_{F}\left(a l_{\sigma}(b)\right)=u^{\varepsilon_{n_{1}} r_{1}-2 \delta_{n_{1}+1} m_{1}} v^{2\left(1-n_{1}\right)} x B^{m_{1}-\delta_{n_{1}}}\left(B^{\varepsilon_{n_{1}}} u^{-\varepsilon_{n_{1}}}\right)^{2 \varepsilon_{n_{1}} \delta_{n_{1}} r_{2}} \text {. } \\
& B^{\delta_{n_{1}}\left(1-2 r_{2}\right)-m_{1}} \theta\left(m_{1}+2 \delta_{n_{1}} r_{2}, \delta_{n_{1}}\right)(\rho(y)) \\
& =u^{\varepsilon_{n_{1}} r_{1}-2 \delta_{n_{1}+1} m_{1}-2 \delta_{n_{1}} r_{2}} v^{2\left(1-n_{1}\right)} \cdot v^{2\left(n_{1}-1\right)} u^{2 \delta_{n_{1}} r_{2}} v^{2\left(1-n_{1}\right)} u^{-2 \delta_{n_{1}} r_{2}} \text {. } \\
& u^{2 \delta_{n_{1}} r_{2}} x u^{-2 \delta_{n_{1}} r_{2}} \cdot u^{2 \delta_{n_{1}} r_{2}} B^{m_{1}-\delta_{n_{1}}} u^{-2 \delta_{n_{1}} r_{2}} \cdot u^{2 \delta_{n_{1}} r_{2}} . \\
& \left(B^{\varepsilon_{n_{1}}} u^{-\varepsilon_{n_{1}}}\right)^{2 \varepsilon_{n_{1}} \delta_{n_{1}} r_{2}} B_{0,0}^{\delta_{n_{1}}\left(1-2 r_{2}\right)-m_{1}} \cdot \theta\left(m_{1}+2 \delta_{n_{1}} r_{2}, \delta_{n_{1}}\right)(\rho(y)) \\
& =u^{\varepsilon_{n_{1}} r_{1}-2 \delta_{n_{1}+1} m_{1}-2 \delta_{n_{1}} r_{2}} v^{2\left(1-n_{1}\right)} O_{n_{1}-1,2 \delta_{n_{1}} r_{2}} c_{0,2 \delta_{n_{1}} r_{2}}(x) B_{0,2 \delta_{n_{1}} r_{2}}^{m_{1}-\delta_{n_{1}}} \text {. } \\
& T_{2 \delta_{n_{1}} r_{2}, \delta_{n_{1}}} B_{0,0}^{\delta_{n_{1}}\left(1-2 r_{2}\right)-m_{1}} \theta\left(m_{1}+2 \delta_{n_{1}} r_{2}, \delta_{n_{1}}\right)(\rho(y)) .
\end{aligned}
$$

Applying condition (i) of Lemma 4.1, we see that:

$$
\begin{aligned}
& c_{2\left(n_{1}-1\right), 2 \delta_{n_{1}+1} m_{1}-\varepsilon_{n_{1}} r_{1}}(y) B_{2\left(n_{1}-1\right), 2 \delta_{n_{1}+1} m_{1}-\varepsilon_{n_{1}} r_{1}}^{r_{2}} J_{n_{1}-1,-2 r_{2}} B_{0,0}^{-r_{2}} \theta\left(r_{2}, 0\right)(x)= \\
& O_{n_{1}-1,2 \delta_{n_{1}} r_{2}} c_{0,2 \delta_{n_{1}} r_{2}}(x) B_{0,2 \delta_{n_{1}} r_{2}}^{m_{1}-\delta_{n_{1}}} T_{2 \delta_{n_{1}} r_{2}, \delta_{n_{1}}} B_{0,0}^{\delta_{n_{1}}\left(1-2 r_{2}\right)-m_{1}} \theta\left(m_{1}+2 \delta_{n_{1}} r_{2}, \delta_{n_{1}}\right)(\rho(y)),
\end{aligned}
$$

in $\overline{\left\langle\sigma^{2}\right\rangle}$, and by Abelianising this equation, we obtain the following equality in ${\overline{\left\langle\sigma^{2}\right\rangle}}_{\mathrm{Ab}}$ :

$$
\begin{gathered}
\left(c_{0,2 \delta_{n_{1}} r_{2}}\right)_{\mathrm{Ab}}(x)-\theta\left(r_{2}, 0\right)_{\mathrm{Ab}}(x)+\theta\left(m_{1}+2 \delta_{n_{1}} r_{2}, \delta_{n_{1}}\right)_{\mathrm{Ab}} \circ \rho_{a b}(y)-\left(c_{2\left(n_{1}-1\right), 2 \delta_{n_{1}+1} m_{1}-\varepsilon_{n_{1}} r_{1}}\right)_{\mathrm{Ab}}(y)= \\
\widetilde{J}_{n_{1}-1,-2 r_{2}}-\widetilde{O}_{n_{1}-1,2 \delta_{n_{1}} r_{2}}-\widetilde{T}_{2 \delta_{n_{1}} r_{2}, \delta_{n_{1}}}+r_{2} B_{2\left(n_{1}-1\right), 2 \delta_{n_{1}+1} m_{1}-\varepsilon_{n_{1}} r_{1}} \\
-\left(m_{1}-\delta_{n_{1}}\right) B_{0,2 \delta_{n_{1}} r_{2}}-\left(\delta_{n_{1}}\left(1-2 r_{2}\right)-m_{1}+r_{2}\right) B_{0,0} .
\end{gathered}
$$

Now by (15)-(17), for all $k, l \in \mathbb{Z}$, we may check that:

$$
\begin{aligned}
& \mu\left(B_{k, l}\right)=\left(c_{0,2 \delta_{n_{1}} r_{2}}\right)_{\mathrm{Ab}}\left(B_{k, l}\right)-\theta\left(r_{2}, 0\right)_{\mathrm{Ab}}\left(B_{k, l}\right) \text { and } \\
& \nu\left(B_{k, l}\right)=\theta\left(m_{1}+2 \delta_{n_{1}} r_{2}, \delta_{n_{1}}\right) \mathrm{Ab} \circ \rho_{a b}\left(B_{k, l}\right)-\left(c_{2\left(n_{1}-1\right), 2 \delta_{n_{1}+1} m_{1}-\varepsilon_{n_{1}} r_{1}}\right)_{\mathrm{Ab}}\left(B_{k, l}\right) .
\end{aligned}
$$

Equation (33) then follows from (36), (37) and (38).

In what follows, we suppose that the hypotheses of Proposition 4.7 hold, namely $r_{1} \geq 0, r_{2} \neq 0$, and either $r_{1}=0$, or $r_{1}>0$ and $e\left(r_{1}\right)>e\left(r_{2}\right)$. With the notation of Lemma 4.10, we define the homomorphism $\xi_{n_{1}, r_{2}}:{\overline{\left\langle\sigma^{2}\right\rangle}}_{\mathrm{Ab}} \rightarrow \mathbb{Z}_{2}$ on the basis $\left\{B_{k, l}\right\}_{k, l \in \mathbb{Z}}$ as follows:

$$
\xi_{n_{1}, r_{2}}\left(B_{k, l}\right)= \begin{cases}\overline{0} & \text { if } k \neq n_{1}-1, \text { or if } k=n_{1}-1 \text { and } 2^{e\left(r_{2}\right)+1} \nmid l \\ \overline{1} & \text { if } k=n_{1}-1 \text { and } 2^{e\left(r_{2}\right)+1} \mid l .\end{cases}
$$

Lemma 4.11. With the notation of Lemma 4.10, the compositions $\xi_{n_{1}, r_{2}} \circ \mu:{\overline{\left\langle\sigma^{2}\right\rangle}}_{A b} \rightarrow \mathbb{Z}_{2}$ and $\xi_{n_{1}, r_{2}} \circ \nu:{\overline{\left\langle\sigma^{2}\right\rangle}}_{A b} \rightarrow \mathbb{Z}_{2}$ are identically zero. 
Proof. It suffices to prove that $\xi_{n_{1}, r_{2}} \circ \mu\left(B_{k, l}\right)=\xi_{n_{1}, r_{2}} \circ \nu\left(B_{k, l}\right)=\overline{0}$ for all $k, l \in \mathbb{Z}$. We start with the case of $\xi_{n_{1}, r_{2}} \circ \mu$. By (34), clearly $\xi_{n_{1}, r_{2}} \circ \mu\left(B_{k, l}\right)=\overline{0}$ if $k \neq n_{1}-1$. So suppose that $k=n_{1}-1$. Using the fact that $\varepsilon_{n_{1}-1} \delta_{n_{1}}=\delta_{n_{1}}$, we have:

$$
\begin{aligned}
\xi_{n_{1}, r_{2}} \circ \mu\left(B_{n_{1}-1, l}\right) & =\xi_{n_{1}, r_{2}}\left(B_{n_{1}-1, l+2 \varepsilon_{n_{1}-1} \delta_{n_{1}} r_{2}}-B_{n_{1}-1, l-2 \delta_{n_{1}-1} r_{2}}\right) \\
& =\xi_{n_{1}, r_{2}}\left(B_{n_{1}-1, l+2 \delta_{n_{1}} r_{2}}\right)-\xi_{n_{1}, r_{2}}\left(B_{n_{1}-1, l-2 \delta_{n_{1}-1} r_{2}}\right) .
\end{aligned}
$$

Now $\left(l+2 \delta_{n_{1}} r_{2}\right)-\left(l-2 \delta_{n_{1}-1} r_{2}\right)=2 r_{2}\left(\delta_{n_{1}}+\delta_{n_{1}-1}\right)=2 r_{2}$, hence $2^{e\left(r_{2}\right)+1} \mid l+2 \delta_{n_{1}} r_{2}$ if and only if $2^{e\left(r_{2}\right)+1} \mid l-2 \delta_{n_{1}-1} r_{2}$, and it follows from (39) and (40) that $\xi_{n_{1}, r_{2}} \circ \mu\left(B_{n_{1}-1, l}\right)=\overline{0}$ as required. We now analyse the case of $\xi_{n_{1}, r_{2}} \circ \nu$. Since $-k=n_{1}-1$ if and only if $k+2\left(n_{1}-1\right)=n_{1}-1$, it follows from (35) that $\xi_{n_{1}, r_{2}} \circ \nu\left(B_{k, l}\right)=\overline{0}$ if $k \neq-\left(n_{1}-1\right)$. So suppose that $k=-\left(n_{1}-1\right)$. Since $\varepsilon_{k} \varepsilon_{n_{1}}=\varepsilon_{n_{1}-1} \varepsilon_{n_{1}}=-1, \varepsilon_{n_{1}} \varepsilon_{k+1}=\varepsilon_{n_{1}}^{2}=1, \delta_{n_{1}-1} \delta_{n_{1}}=0$ and $\varepsilon_{n_{1}-1} \delta_{n_{1}+1}=-\delta_{n_{1}+1}$, we obtain:

$$
\begin{aligned}
\xi_{n_{1}, r_{2}} \circ \nu\left(B_{-\left(n_{1}-1\right), l}\right) & =-\xi_{n_{1}, r_{2}}\left(B_{n_{1}-1, l-2 \delta_{n_{1}-1}\left(m_{1}+2 \delta_{n_{1}} r_{2}\right)}\right)-\xi_{n_{1}, r_{2}}\left(B_{n_{1}-1, l+\varepsilon_{n_{1}-1}\left(2 \delta_{n_{1}+1} m_{1}-\varepsilon_{n_{1}} r_{1}\right)}\right) \\
& =-\xi_{n_{1}, r_{2}}\left(B_{n_{1}-1, l-2 \delta_{n_{1}-1} m_{1}}\right)-\xi_{n_{1}, r_{2}}\left(B_{n_{1}-1, l-2 \delta_{n_{1}+1} m_{1}+r_{1}}\right) .
\end{aligned}
$$

Now $\left(l-2 \delta_{n_{1}-1} m_{1}\right)-\left(l-2 \delta_{n_{1}+1} m_{1}+r_{1}\right)=-r_{1}$. Since $2^{e\left(r_{2}\right)+1} \mid r_{1}$, it follows that $2^{e\left(r_{2}\right)+1} \mid$ $l-2 \delta_{n_{1}-1} m_{1}$ if and only if $2^{e\left(r_{2}\right)+1} \mid l-2 \delta_{n_{1}+1} m_{1}+r_{1}$. Equations (39) and (41) then imply that $\xi_{n_{1}, r_{2}} \circ \nu\left(B_{-\left(n_{1}-1\right), l}\right)=\overline{0}$ as required.

We now complete the proof of Theorem 1.3. The following remark will be used in the proof of Proposition 4.7 .

Remark 4.12. Let $r \in \mathbb{Z} \backslash\{0\}$, and let $S$ be a set consisting of $2|r|$ consecutive integers. Then $S$ contains $o(r)=|r| / 2^{e(r)}$ elements divisible by $2^{e(r)+1}$.

of Proposition 4.7. Let $\alpha \in\left[\mathbb{T}^{2}, * ; \mathbb{K}^{2}, *\right]$ be a pointed homotopy class such that $\alpha_{\#}(1,0)=\left(r_{1}, 2\right)$ and $\alpha_{\#}(0,1)=\left(r_{2}, 0\right)$, where $r_{2} \neq 0$ and either $r_{1}=0$, or $r_{1}>0$ and $e\left(r_{1}\right)>e\left(r_{2}\right)$. Suppose on the contrary that $\alpha$ does not have the Borsuk-Ulam property with respect to $\tau_{1}$. Then by Lemma 4.10, there exist $x, y \in{\overline{\left\langle\sigma^{2}\right\rangle}}_{\mathrm{Ab}}$ such that equation (33) holds. By Lemma 4.11, $\xi_{n_{1}, r_{2}}(\mu(x)+\nu(y))=\overline{0}$. So to prove the result, it suffices to show that the image of the right-hand side of (33) by $\xi_{n_{1}, r_{2}}$ is equal to $\overline{1}$. We analyse each of the terms in turn.

(a) We start by showing that:

$$
\xi_{n_{1}, r_{2}}\left(\widetilde{J}_{n_{1}-1,-2 r_{2}}\right)=\delta_{n_{1}+1} \overline{1} .
$$

If $n_{1}=1$ then $\widetilde{J}_{n_{1}-1,-2 r_{2}}=0$ by Proposition $3.7(\mathrm{~b})$, so $\xi_{n_{1}, r_{2}}\left(\widetilde{J}_{n_{1}-1,-2 r_{2}}\right)=\overline{0}$. So suppose that $n_{1} \neq 1$. By Proposition 3.7(c) and (39), we have:

$$
\xi_{n_{1}, r_{2}}\left(\widetilde{J}_{n_{1}-1,-2 r_{2}}\right)=\sum_{i=1}^{\sigma_{n_{1}-1}\left(n_{1}-1\right)} \sum_{j=1}^{\sigma_{r_{2}} 2 r_{2}} \xi_{n_{1}, r_{2}}\left(B_{\sigma_{n_{1}-1}(2 i-1),-\sigma_{2 r_{2}}\left(j-\left(1-\sigma_{2 r_{2}}\right) / 2\right)}\right) .
$$

If $n_{1}$ is odd, there is no integer $i$ satisfying $\sigma_{n_{1}-1}(2 i-1)=n_{1}-1$, and thus $\xi_{n_{1}, r_{2}}\left(\widetilde{J}_{n_{1}-1,-2 r_{2}}\right)=\overline{1}$ in this case. If $n_{1}$ is even, then $\sigma_{n_{1}-1}(2 i-1)=n_{1}-1$ if and only if $i=\left(\sigma_{n_{1}-1}\left(n_{1}-1\right)+\right.$ $1) / 2$, and in this case, $i$ belongs to the allowed set $\left\{1, \ldots, \sigma_{n_{1}-1}\left(n_{1}-1\right)\right\}$ of indices. Now consider the terms of the form $B_{n_{1}-1,-\sigma_{2 r_{2}}\left(j-\left(1-\sigma_{2 r_{2}}\right) / 2\right)}$, where $j \in\left\{1, \ldots, \sigma_{r_{2}} 2 r_{2}\right\}$. Then the set $\left\{-\sigma_{2 r_{2}}\left(j-\left(1-\sigma_{2 r_{2}}\right) / 2\right) \mid j=1, \ldots, \sigma_{r_{2}} 2 r_{2}\right\}$ consists of $2\left|r_{2}\right|$ consecutive integers, and thus contains $o\left(r_{2}\right)$ elements divisible by $2^{e\left(r_{2}\right)+1}$ by Remark 4.12. It follows from (39) that $\xi_{n_{1}, r_{2}}\left(\widetilde{J}_{n_{1}-1,-2 r_{2}}\right)=\overline{1}$, and this proves $(42)$. 
(b) Consider the term $\widetilde{O}_{n_{1}-1,2 \delta_{n_{1}} r_{2}}$. If $n_{1}$ is even or is equal to 1 then $\widetilde{O}_{n_{1}-1,2 \delta_{n_{1}} r_{2}}=0$ by Proposition 3.7(b), and $\xi_{n_{1}, r_{2}}\left(\widetilde{O}_{n_{1}-1,2 \delta_{n_{1}} r_{2}}\right)=\overline{0}$. So assume that $n_{1}$ is odd and different from 1. By Proposition 3.7(c) and (39), we have:

$$
\begin{aligned}
\xi_{n_{1}, r_{2}}\left(\widetilde{O}_{n_{1}-1,2 \delta_{n_{1}} r_{2}}\right)= & \xi_{n_{1}, r_{2}}\left(\widetilde{O}_{n_{1}-1,2 r_{2}}\right) \\
= & \sum_{i=1}^{\sigma_{n_{1}-1}\left(n_{1}-1\right)} \sum_{j=1}^{\sigma_{r_{2}} 2 r_{2}}\left(\xi_{n_{1}, r_{2}}\left(B_{\sigma_{n_{1}-1}(2 i-1),-\sigma_{2 r_{2}} j+\left(\sigma_{2 r_{2}}-1\right) / 2}\right)+\right. \\
& \left.\xi_{n_{1}, r_{2}}\left(B_{\sigma_{n_{1}-1}(2 i-1)-1, \sigma_{2 r_{2}} j-\left(\sigma_{2 r_{2}}+1\right) / 2}\right)\right) .
\end{aligned}
$$

Observe that there is no integer $i$ satisfying $\sigma_{n_{1}-1}(2 i-1)=n_{1}-1$, and it follows that $\xi_{n_{1}, r_{2}}\left(B_{\sigma_{n_{1}-1}(2 i-1),-\sigma_{2 r_{2}} j+\left(\sigma_{2 r_{2}}-1\right) / 2}\right)=\overline{0}$ for all $i \in\left\{1, \ldots, \sigma_{n_{1}-1}\left(n_{1}-1\right)\right\}$ and $j \in\left\{1, \ldots, \sigma_{r_{2}} 2 r_{2}\right\}$. For the second term of $(43)$, note that $\sigma_{n_{1}-1}(2 i-1)-1=n_{1}-1$ if and only if $i=$ $\left(\sigma_{n_{1}-1}\left(n_{1}-1\right)+\sigma_{n_{1}-1}+1\right) / 2$, and in this case, $i$ belongs to the allowed set $\left\{1, \ldots, \sigma_{n_{1}-1}\left(n_{1}-1\right)\right\}$ of indices. Now consider the terms of the form $B_{n_{1}-1, \sigma_{2 r_{2}} j-\left(\sigma_{2 r_{2}}-1\right) / 2}$, where $j \in\left\{1, \ldots, \sigma_{r_{2}} 2 r_{2}\right\}$. Then the set $\left\{\sigma_{2 r_{2}} j-\left(\sigma_{2 r_{2}}+1\right) / 2 \mid j=1, \ldots, \sigma_{r_{2}} 2 r_{2}\right\}$ consists of $2\left|r_{2}\right|$ consecutive integers, and thus contains $o\left(r_{2}\right)$ elements divisible by $2^{e\left(r_{2}\right)+1}$ by Remark 4.12. It follows from (39) that $\xi_{n_{1}, r_{2}}\left(\widetilde{O}_{n_{1}-1,2 r_{2}}\right)=\overline{1}$. Hence:

$$
\xi_{n_{1}, r_{2}}\left(\widetilde{O}_{n_{1}-1,2 \delta_{n_{1}} r_{2}}\right)=\delta_{n_{1}} \overline{1}
$$

(c) Consider the term $\widetilde{T}_{2 \delta_{n_{1}} r_{2}, \delta_{n_{1}}}$. If $n_{1}$ is even then $\widetilde{T}_{2 \delta_{n_{1}} r_{2}, \delta_{n_{1}}}=0$ by Proposition 3.7(b), and thus $\xi_{n_{1}, r_{2}}\left(\widetilde{T}_{2 \delta_{n_{1}} r_{2}, \delta_{n_{1}}}\right)=\overline{0}$. So assume that $n_{1}$ is odd. By Proposition 3.7(c), we have:

$$
\widetilde{T}_{2 \delta_{n_{1}} r_{2}, \delta_{n_{1}}}=\widetilde{T}_{2 r_{2}, 1}=\sigma_{r_{2}} \sum_{i=1}^{\sigma_{r_{2}} 2 r_{2}} B_{0, \sigma_{r_{2}}\left(i-\left(\sigma_{r_{2}}+1\right) / 2\right)} .
$$

If $n_{1} \neq 1$ then $\xi_{n_{1}, r_{2}}\left(\widetilde{T}_{2 r_{2}, 1}\right)=\overline{0}$ by (39). So suppose that $n_{1}=1$. Then the set $\left\{\sigma_{r_{2}}\left(i-\left(\sigma_{r_{2}}+\right.\right.\right.$ 1)/2) $\left.\mid i=1, \ldots, \sigma_{r_{2}} 2 r_{2}\right\}$ of indices consists of $2\left|r_{2}\right|$ consecutive integers, and thus contains $o\left(r_{2}\right)$ elements divisible by $2^{e\left(r_{2}\right)+1}$ by Remark 4.12. It follows from (39) that $\xi_{n_{1}, r_{2}}\left(\widetilde{T}_{2 r_{2}, 1}\right)=\overline{1}$. Hence:

$$
\xi_{n_{1}, r_{2}}\left(\widetilde{T}_{2 \delta_{n_{1}} r_{2}, \delta_{n_{1}}}\right)= \begin{cases}\overline{1} & \text { if } n_{1}=1 \\ \overline{0} & \text { otherwise. }\end{cases}
$$

(d) Let $\chi=r_{2} B_{2\left(n_{1}-1\right), 2 \delta_{n_{1}+1} m_{1}-\varepsilon_{n_{1}} r_{1}}-\left(m_{1}-\delta_{n_{1}}\right) B_{0,2 \delta_{n_{1}} r_{2}}-\left(\delta_{n_{1}}\left(1-2 r_{2}\right)-m_{1}+r_{2}\right) B_{0,0}$. If $n_{1} \neq 1$ then it follows from (39) that $\xi_{n_{1}, r_{2}}(\chi)=\overline{0}$. So suppose that $n_{1}=1$. Then $\chi=$ $r_{2} B_{0, r_{1}}-\left(m_{1}-1\right) B_{0,2 r_{2}}-\left(1-m_{1}-r_{2}\right) B_{0,0}$. By hypothesis, $e\left(r_{1}\right)>e\left(r_{2}\right)$, and we see from (39) that $\xi_{n_{1}, r_{2}}(\chi)=\overline{r_{2}}+\overline{m_{1}-1}+\overline{1-m_{1}-r_{2}}=\overline{0}$. Thus:

$$
\begin{aligned}
\xi_{n_{1}, r_{2}}\left(r_{2} B_{2\left(n_{1}-1\right), 2 \delta_{n_{1}+1} m_{1}-\varepsilon_{n_{1}} r_{1}}-\left(m_{1}-\delta_{n_{1}}\right) B_{0,2 \delta_{n_{1}} r_{2}}-\right. & \\
& \left.\left(\delta_{n_{1}}\left(1-2 r_{2}\right)-m_{1}+r_{2}\right) B_{0,0}\right)=\overline{0} .
\end{aligned}
$$

We now take the image of (33) by $\xi_{n_{1}, r_{2}}$. Using (47) and Lemma 4.10, it follows that:

$$
\overline{0}=\xi_{n_{1}, r_{2}}(\mu(x)+\nu(y))=\xi_{n_{1}, r_{2}}\left(\widetilde{J}_{n_{1}-1,-2 r_{2}}-\widetilde{O}_{n_{1}-1,2 \delta_{n_{1}} r_{2}}-\widetilde{T}_{2 \delta_{n_{1}} r_{2}, \delta_{n_{1}}}\right) .
$$

From (42), (44) and (45), we obtain the following conclusions: 
(i) if $n_{1}$ is even then $\xi_{n_{1}, r_{2}}\left(\widetilde{J}_{n_{1}-1,-2 r_{2}}\right)=\overline{1}$ and $\xi_{n_{1}, r_{2}}\left(\widetilde{O}_{n_{1}-1,2 \delta_{n_{1}} r_{2}}\right)=\xi_{n_{1}, r_{2}}\left(\widetilde{T}_{2 \delta_{n_{1}} r_{2}, \delta_{n_{1}}}\right)=\overline{0}$.

(ii) if $n_{1}=1$ then $\xi_{n_{1}, r_{2}}\left(\widetilde{T}_{2 \delta_{n_{1}} r_{2}, \delta_{n_{1}}}\right)=\overline{1}$ and $\xi_{n_{1}, r_{2}}\left(\widetilde{O}_{n_{1}-1,2 \delta_{n_{1}} r_{2}}\right)=\xi_{n_{1}, r_{2}}\left(\widetilde{J}_{n_{1}-1,-2 r_{2}}\right)=\overline{0}$.

(iii) if $n_{1}$ is odd and $n_{1} \neq 1$ then $\xi_{n_{1}, r_{2}}\left(\widetilde{J}_{n_{1}-1,-2 r_{2}}\right)=\xi_{n_{1}, r_{2}}\left(\widetilde{T}_{2 \delta_{n_{1}} r_{2}, \delta_{n_{1}}}\right)=\overline{0}$ and $\xi_{n_{1}, r_{2}}\left(\widetilde{O}_{n_{1}-1,2 \delta_{n_{1}} r_{2}}\right)=$ $\overline{1}$.

In all three cases, we conclude that $\xi_{n_{1}, r_{2}}\left(\widetilde{J}_{n_{1}-1,-2 r_{2}}-\widetilde{O}_{n_{1}-1,2 \delta_{n_{1}} r_{2}}-\widetilde{T}_{2 \delta_{n_{1}} r_{2}, \delta_{n_{1}}}\right)=\overline{1}$, which contradicts equation (48). It follows that $\alpha$ has the Borsuk-Ulam property with respect to $\tau_{1}$.

\section{A Appendix}

Let $g: F(u, v) \rightarrow \mathbb{Z} \rtimes \mathbb{Z}$ be the homomorphism defined on the generators of $F(u, v)$ by $g(u)=(1,0)$ and $g(v)=(0,1)$.

Proposition A.1. For each $k, l \in \mathbb{Z}, l \neq 0$, let $\Gamma_{k, l}=v^{k} u^{l} v u^{l} v^{-k-1} \in F(u, v)$. Then $\operatorname{Ker}(g)=$ $\left\langle\Gamma_{k, l}, k, l \in \mathbb{Z}, l \neq 0 \mid-\right\rangle$.

Proof. We use the Reidemeister-Schreier rewriting process that is described in detail in [8, Chapter 2, Theorem 2.8] and briefly in [10, Appendix I, Theorem 6.3]. We use the notation of [10]. Let $S=\left\{v^{k} u^{l}\right\}_{k, l \in \mathbb{Z}}$. We have $g\left(v^{k} u^{l}\right)=(0, k)(l, 0)=\left((-1)^{k} l, k\right)$. So, $\left.g\right|_{S}: S \rightarrow \mathbb{Z} \rtimes \mathbb{Z}$ is a bijection, and therefore $S$ is a complete set of right coset representatives of $\operatorname{Ker}(g)$ in $F(u, v)$. Moreover, $S$ is a Schreier system of $\operatorname{Ker}(g)$. Let us compute the generators of $\operatorname{Ker}(g)$. We have

$$
\begin{aligned}
& v^{k} u^{l} u{\overline{v^{k} u^{l} u}}^{-1}=v^{k} u^{l+1}{\overline{v^{k} u^{l+1}}}^{-1}=v^{k} u^{l+1}\left(v^{k} u^{l+1}\right)^{-1}=\mathbf{1}, \text { and } \\
& v^{k} u^{l} v{\overline{v^{k} u^{l} v}}^{-1}=v^{k} u^{l} v\left(v^{k+1} u^{-l}\right)^{-1}=\Gamma_{k, l},
\end{aligned}
$$

where for all $w \in F(u, v), \bar{w}$ is the unique element of $S$ for which $g(\bar{w})=g(w)$. Note that $\Gamma_{k, l}=\mathbf{1}$ if and only if $l=0$. Using the Reidemeister-Schreier rewriting process, we see that the group $\operatorname{Ker}(g)$ is freely generated by $\left\{\Gamma_{k, l}\right\}_{k, l \in \mathbb{Z}, l \neq 0}$.

The basis of $\operatorname{Ker}(g)$ given in Proposition A.1 is not well adapted to our calculations. We define a new basis that is more suitable.

Lemma A.2. For each $k, l \in \mathbb{Z}$, let $B_{k, l}=v^{k} u^{l} B u^{-l} v^{-k}$, where $B=u v u v^{-1}$. Then we have the following relations in $\operatorname{Ker}(g)$ :

(a) $\Gamma_{k, l}=\prod_{i=1}^{l} B_{k, l-i}$ if $l \geq 1$, and $\Gamma_{k, l}=\prod_{i=1}^{-l} B_{k, l-1+i}^{-1}$ if $l \leq-1$.

(b) $B_{k, l}=\Gamma_{k, l+1} \Gamma_{k, l}^{-1}$, where $\Gamma_{k, 0}^{-1}=\mathbf{1}$.

Proof. We first prove part (a). We start by proving the result in the case $k=0$. If $l=1$, we have $\Gamma_{0,1}=u v u v^{-1}=B_{0,0}$. So suppose that $\Gamma_{0, l}=\prod_{i=1}^{l} B_{0, l-i}$ for some $l \geq 1$, and let us show that the result holds for $l+1$. We have:

$$
\Gamma_{0, l+1}=u \Gamma_{0, l} u^{-1} \Gamma_{0,1}=u\left(\prod_{i=1}^{l} B_{0, l-i}\right) u^{-1} B_{0,0}=\left(\prod_{i=1}^{l} B_{0, l-i+1}\right) B_{0,0}=\prod_{i=1}^{l+1} B_{0,(l+1)-i} .
$$


By induction, the given formula is valid for $k=0$ and all $l \geq 1$. If $l \leq-1$, the result holds for $\Gamma_{0,-l}$, and thus:

$$
\Gamma_{0, l}=u^{l} \Gamma_{0,-l}^{-1} u^{-l}=u^{l}\left(\prod_{i=1}^{-l} B_{0,-l-i}\right)^{-1} u^{-l}=\left(\prod_{i=1}^{-l} B_{0,-i}\right)^{-1}=\prod_{i=1}^{-l} B_{0, l-1+i}^{-1} .
$$

Hence the formula holds for $k=0$ and all $l \in \mathbb{Z} \backslash\{0\}$. Now let $k \in \mathbb{Z}$. Then $\Gamma_{k, l}=v^{k} \Gamma_{0, l} v^{-k}$ and $B_{k, l}=v^{k} B_{0, l} v^{-k}$, and we obtain the formula for all $k \in \mathbb{Z}$ and $l \in \mathbb{Z} \backslash\{0\}$ using the results of the case $k=0$. Part (b) then follows.

Theorem A.3. The set $\left\{B_{k, l}=v^{k} u^{l} B u^{-l} v^{-k}\right\}_{k, l \in \mathbb{Z}}$ is a basis of $\operatorname{Ker}(g)$.

Proof. By Lemma A.2, the elements of the set $\left\{B_{k, l}\right\}_{k, l \in \mathbb{Z}}$ generate $\operatorname{Ker}(g)$. To show that this set is a basis, it suffices to prove that there are only trivial relations between these elements. Suppose on the contrary that there exists a word $w \in \operatorname{Ker}(g)$ for which:

$$
w=B_{k_{1}, l_{1}}^{\varepsilon 1} B_{k_{2}, l_{2}}^{\varepsilon_{2}} \cdots B_{k_{n}, l_{n}}^{\varepsilon_{n}}=\mathbf{1}, \text { where } \varepsilon_{i} \in\{-1,1\} \text { and } B_{k_{i}, r_{i}}^{\varepsilon_{i}} B_{k_{i+1}, r_{i+1}}^{\varepsilon_{i+1}} \neq \mathbf{1}
$$

Let $S=\left\{k_{1}, k_{2}, \ldots, k_{n}\right\}$. For each $k \in S$, we define the set $R_{k}$ consisting of those indices $l_{j}$ for which the element $B_{k, l_{j}}$ appears in the word $w$. Let $l_{m}$ and $l_{M}$ be the minimal and maximal elements of $R_{k}$ respectively. We define the sets $B_{k}$ and $\Gamma_{k}$ as follows:

- $B_{k}=\left\{B_{k, 0}, \ldots, B_{k, l_{m}}, \ldots, B_{k, l_{M}}\right\}$ and $\Gamma_{k}=\left\{\Gamma_{k, 1} \ldots, \Gamma_{k, l_{M}}, \Gamma_{k, l_{M}+1}\right\}$ if $0 \leq l_{m}$.

- $B_{k}=\left\{B_{k, l_{m}}, \ldots, B_{k,-1}, B_{k, 0}, B_{k, 1}, \ldots, B_{k, l_{M}}\right\}$ and $\Gamma_{k}=\left\{\Gamma_{k, l_{m}}, \ldots, \Gamma_{k,-1}, \Gamma_{k, 1}, \ldots, \Gamma_{k, l_{M}+1}\right\}$ if $l_{m}<0<l_{M}+1$.

- $B_{k}=\left\{B_{k, l_{m}}, \ldots, B_{k, l_{M}}\right\}$ and $\Gamma_{k}=\left\{\Gamma_{k, l_{m}}, \ldots, \Gamma_{k, l_{M}}\right\}$ if $l_{M}+1=0$.

- $B_{k}=\left\{B_{k, l_{m}}, \ldots, B_{k, l_{M}}, B_{k, l_{M}+1}\right\}$ and $\Gamma_{k}=\left\{\Gamma_{k, l_{m}}, \ldots, \Gamma_{k, l_{M}}, \Gamma_{k, l_{M}+1}\right\}$ if $l_{M}+1<0$.

Note that $B_{k}$ and $\Gamma_{k}$ have the same number of elements by Lemma A.2. Further, if $k, k^{\prime} \in S$, where $k \neq k^{\prime}$, then $B_{k} \cap B_{k^{\prime}}=\emptyset=\Gamma_{k} \cap \Gamma_{k^{\prime}}$. It follows that $C_{B}=\bigcup_{k \in S} B_{s}$ and $C_{\Gamma}=\bigcup_{k \in S} \Gamma_{k}$ have

the same number of elements, and generate the same subgroup $C$ of $\operatorname{Ker}(g)$, using Lemma A.2 once more. Since $C_{\Gamma}$ is a finite basis of $C, C_{B}$ is a basis of $C$, and so $w \in C$, which yields a contradiction because $C$ is a free group.

\section{Acknowledgements}

This work is a continuation of part of the Ph.D. thesis [7] of the third author who was supported by CNPq project $\mathrm{n}^{\mathrm{O}} 140836$ and Capes/COFECUB project $\mathrm{n}^{\circ} 12693 / 13-8$. The first and second authors wish to thank the 'Réseau Franco-Brésilien en Mathématiques' for financial support during their respective visits to the Laboratoire de Mathématiques Nicolas Oresme UMR CNRS 6139, Université de Caen Normandie, from the $9^{\text {th }}$ to the $24^{\text {th }}$ of November 2019, and to the Instituto de Matemática e Estatística, Universidade de São Paulo, from the $17^{\text {th }}$ to the $31^{\text {th }}$ of August 2019. 


\section{References}

[1] K. Bonsuk, Drei Sätze über die n-dimensionale Euklidische Sphäre, Fund. Math. 20 (1933), 177-190.

[2] E. Fadell, L. Neuwirth, Configuration spaces, Math. Scand. 10 (1962), 111-118.

[3] D. L. GonçALves, The Borsuk-Ulam theorem for surfaces, Quaest. Math. 29 (2006), $117-$ 123.

[4] D. L. Gonçalves, J. Guaschi, The Borsuk-Ulam theorem for maps into a surface, Top. Appl. 157 (2010), 1742-1759.

[5] D. L. Gonçalves, J. Guaschi, V. C. LaAss, The Borsuk-Ulam property for homotopy classes of selfmaps of surfaces of Euler characteristic zero, J. Fixed Point Theory Appl. 21 (2019) 65.

[6] D. L. Gonçalves, M. R. Kelly, Coincidence properties for maps from the torus to the Klein bottle, Chin. Ann. Math. 29B (2008), 425-440.

[7] V. C. LaAss, A propriedade de Borsuk-Ulam para funções entre superfícies, Ph.D Thesis, IME, Universidade de São Paulo (2015).

[8] W. Magnus, A. Karrass, D. Solitar, Combinatorial group theory: presentations of groups in terms of generators and relations, 2nd edition, Dover Publications, Inc., Mineola, NY, (2004).

[9] J. Matoušek, Using the Borsuk-Ulam theorem, Universitext, Springer-Verlag (2002).

[10] K. Murasugi, B. I. Kurpita, A study of braids, Mathematics and Its Applications 484, Kluwer Academic Publishers, (1999).

[11] G. W. Whitenead, Elements of homotopy theory, Graduate Texts in Mathematics 61, Springer-Verlag (1978).

[12] H. Zieschang, E. Vogt, H.-D. Coldewey, Surfaces and planar discontinuous groups, Lecture Notes in Mathematics 835, Springer-Verlag (1980). 